\title{
Constraining the thermally pulsing asymptotic giant branch phase with resolved stellar populations in the Large Magellanic Cloud
}

Giada Pastorelli ${ }^{1,2},{ }^{\star}$ Paola Marigo $^{2}$, Léo Girardi ${ }^{3}$, Bernhard Aringer $^{2}$, Yang Chen $^{2}$, Stefano Rubele ${ }^{2,3}$, Michele Trabucchi ${ }^{9,2}$, Sara Bladh ${ }^{1,4}$, Martha L. Boyer ${ }^{1}$, Alessandro Bressan ${ }^{5}$, Julianne J. Dalcanton ${ }^{6}$, Martin A.T. Groenewegen ${ }^{7}$, Thomas Lebzelter $^{8}$, Nami Mowlavi ${ }^{9}$, Katy L. Chubb ${ }^{10}$, Maria-Rosa L. Cioni1 ${ }^{11}$, Richard de Grijs ${ }^{12,13,14}$, Valentin D. Ivanov ${ }^{15}$, Ambra Nanni ${ }^{16}$, Jacco Th. van Loon ${ }^{17}$, and Simone Zaggia ${ }^{3}$

1 STSCI, 3700 San Martin Drive, Baltimore, MD 21218, USA

2 Dipartimento di Fisica e Astronomia Galileo Galilei, Università di Padova, Vicolo dell'Osservatorio 3, I-35122 Padova, Italy

3 Osservatorio Astronomico di Padova - INAF, Vicolo dell'Osservatorio 5, I-35122 Padova, Italy

4 Theoretical Astrophysics, Department of Physics and Astronomy, Uppsala University, Box 516, SE-751 20 Uppsala, Sweden

5 SISSA, via Bonomea 365, I-34136 Trieste, Italy

6 Department of Astronomy, University of Washington, Box 351580, Seattle, WA 98195, USA

7 Koninklijke Sterrenwacht van België, Ringlaan 3, 1180 Brussel, Belgium

8 University of Vienna, Department of Astrophysics, Tuerkenschanzstrasse 17, A1180 Vienna, Austria

9 Department of Astronomy, University of Geneva, Ch. des Maillettes 51, 1290 Versoix, Switzerland

10 SRON Netherlands Institute for Space Research, Sorbonnelaan 2, 3584 CA, Utrecht, Netherlands

11 Leibniz-Institüt für Astrophysik Potsdam, An der Sternwarte 16, D-14482 Potsdam, Germany

12 Department of Physics and Astronomy, Macquarie University, Balaclava Road, Sydney, NSW 2109, Australia

13 Research Centre for Astronomy, Astrophysics and Astrophotonics, Macquarie University, Balaclava Road, Sydney, NSW 2109, Australia

14 International Space Science Institute-Beijing, 1 Nanertiao, Zhongguancun, Hai Dian District, Beijing 100190, China

15 European Southern Observatory, Karl-Schwarzschild-Str. 2, D-85748 Garching bei MÃijnchen, Germany

16 Aix Marseille UniversitÃl', CNRS, CNES, LAM, 38, rue FrÃl'dÃl'ric Joliot-Curie, F-13388 Marseille, Cedex 13 France

17 Lennard-Jones Laboratories, Keele University, ST5 5BG, UK

\begin{abstract}
Reliable models of the thermally pulsing asymptotic giant branch (TP-AGB) phase are of critical importance across astrophysics, including our interpretation of the spectral energy distribution of galaxies, cosmic dust production, and enrichment of the interstellar medium. With the aim of improving sets of stellar isochrones that include a detailed description of the TP-AGB phase, we extend our recent calibration of the AGB population in the Small Magellanic Cloud (SMC) to the more metal rich Large Magellanic Cloud (LMC). We model the LMC stellar populations with the TRILEGAL code, using the spatially-resolved star formation history derived from the VISTA survey. We characterize the efficiency of the third dredge-up by matching the star counts and the $K_{\mathrm{s}}$-band luminosity functions of the AGB stars identified in the LMC. In line with previous findings, we confirm that, compared to the SMC, the third dredge-up in AGB stars of the LMC is somewhat less efficient, as a consequence of the higher metallicity. The predicted range of initial mass of C-rich stars is between $M_{\mathrm{i}} \approx 1.7-3 \mathrm{M}_{\odot}$ at $Z_{\mathrm{i}}=0.008$. We show how the inclusion of new opacity data in the carbon star spectra will improve the performance of our models. We discuss the predicted lifetimes, integrated luminosities and mass-loss rate distributions of the calibrated models. The results of our calibration are included in updated stellar isochrones publicly available.
\end{abstract}

Key words: stars: AGB and post-AGB - stars: evolution - Magellanic Clouds 


\section{INTRODUCTION}

Close to the end of their lives, low- and intermediatemass stars, with initial masses between approximately 0.8 $\mathrm{M}_{\odot}$ up to 6-8 $\mathrm{M}_{\odot}$, evolve through the thermally pulsing asymptotic giant branch (TP-AGB) phase (Herwig 2005). Despite the very short duration of this evolutionary phase (less than a few Myr), TP-AGB stars can contribute significantly to the integrated luminosity of intermediate-age stellar populations, and the treatment of the TP-AGB phase can affect the interpretation of the spectral energy distribution of unresolved galaxies, in particular the derivation of their stellar mass and age (Maraston et al. 2006; Conroy 2013; Zibetti et al. 2013; Villaume et al. 2015). Furthermore, TP-AGB stars might be significant dust producers in the local Universe and at high redshift (for extensive discussions see e.g. Boyer et al. 2012; Nanni et al. 2018; Schneider et al. 2014; Srinivasan et al. 2016; Valiante et al. 2009; Zhukovska et al. 2008; Zhukovska \& Henning 2013), and can be important contributors to the chemical enrichment of galaxies (Kobayashi et al. 2011; Karakas \& Lattanzio 2014).

TP-AGB stars are also useful to probe the star formation history in other galaxies when deep photometry is not available or not possible, as shown by Javadi et al. (2013, 2017); Rezaeikh et al. (2014); Hamedani Golshan et al. (2017); Hashemi et al. (2019) for M33 and other Local Group galaxies. TP-AGB stars might also offer an additional way to improve the calibration of the extragalactic distance scale either using their long period variability (Pierce et al. 2000; Huang et al. 2018, 2020) or the mean photometric properties of the carbon stars (Ripoche et al. 2020; Madore \& Freedman 2020).

Despite their widespread importance, TP-AGB models suffer from large uncertainties and present models from various authors differ significantly in many important outcomes, such as stellar lifetimes, initial mass of carbon stars, and chemical yields. The main sources of uncertainties can be traced to i) the lack of a robust theory of convection, which affects mixing processes such as third dredge-up (3DU) and hot bottom burning (HBB), and ii) the difficulty of modelling the physics of stellar winds, and therefore the massloss rate as a function of the stellar parameters, which control the TP-AGB lifetimes (Marigo 2015).

In recent years significant progress has been made on both theoretical and observational sides. Now we have the possibility to exploit complete samples of resolved AGB stars from the optical to the infrared wavelengths, observed in stellar systems that span a wide range of metallicities, from the metal poor dwarf galaxies (Dalcanton et al. 2009, 2012; Boyer et al. 2015a, 2017) to the metal rich M31 (Boyer et al. 2019, and Goldman et al., in prep.), and for which we have robust measurements of the star formation history (SFH) (e.g. Weisz et al. 2014; Lewis et al. 2015; Williams et al. 2017)

One fundamental laboratory to study AGB star populations can be found in the Small and Large Magellanic Clouds (SMC and LMC). The stellar populations of these two irregular galaxies are very well studied, and thanks to the numerous spectroscopic and photometric surveys carried out in recent years, we have a complete sample of AGB stars for which we also have a reliable identification of their chemical type, i.e. carbon-rich (C-rich) and oxygen-rich (O-rich).

On the theoretical side, so-called 'full stellar models' are calculated by integrating the stellar structure equations across the whole star, hence resolving the physical structure from the centre to the surface. Moreover, full models still need to rely on parametrized descriptions of complicated three-dimensional processes like convection, overshoot, mass loss. Predictions for the TP-AGB, in particular, are significantly affected by numerical details that may differ from author to author (e.g. this is the case of the third dredgeup; Frost \& Lattanzio 1996). Furthermore, the calculation of full models is time consuming, which makes it difficult to efficiently explore and test the wide range of parameters necessary to provide a thorough calibration of the uncertain processes.

In this context, a complementary approach is provided by the so-called 'envelope models', for which the TP-AGB evolution is calculated by including analytical prescriptions (derived from full model calculations), complemented with envelope integrations. In this work we use the COLIBRI code, fully described in Marigo et al. (2013). COLIBRI combines a synthetic module that includes the freeparameters to be calibrated with the aid of observations, i.e. mass loss, and 3DU occurrence and efficiency, coupled with a complete envelope integration of the stellar structure equations from the atmosphere down to the bottom of the hydrogen-burning shell. This allows the code to follow the changes in the envelope and atmosphere structures (e.g. driven by chemical composition changes) with the same level of detail as in full models, but with a computational time that is typically two orders of magnitude shorter (see e.g. figure 10 of Marigo et al. 2013). This feature is fundamental to efficiently explore the range of parameters that need to be calibrated as a function of stellar mass and metallicity.

By combining the computational agility of the COLIBRI code, and the detailed stellar population synthesis simulations produced with the TRILEGAL code (Girardi et al. 2005), we can test different mass-loss prescriptions and put quantitative constraints on the occurrence and efficiency of the 3DU. This is achieved by reproducing the star counts and the luminosity functions (LFs) of an observed sample of AGB stars with known SFH. The approach was pioneered by Groenewegen \& de Jong (1993), Marigo et al. (1999) and Marigo \& Girardi (2007), and more recently adopted by Girardi et al. (2010) and Rosenfield et al. (2014) using AGB samples in dwarf galaxies from the ANGST survey. In a recent paper, Pastorelli et al. (2019, hereafter Paper I) applied the same approach to the population of AGB stars in the SMC classified by Boyer et al. (2011, hereafter B11) and Srinivasan et al. (2016). The initial metallicity range covered by such work is below $Z_{i}=0.008$.

In this work, we extend the calibration of our TPAGB models to higher metallicities using the observed sample of AGB stars classified by B11 from the Spitzer program 'Surveying the Agents of a Galaxy's Evolution in the LMC' (SAGE-LMC, Blum et al. 2006; Meixner et al. 2006). Starting from the best-fitting model for the SMC, we compute additional evolutionary tracks with initial metallicity $Z_{\mathrm{i}} \geq 0.008$, with the aim of reproducing at the same time the star counts and the luminosity functions of the whole TP-AGB population, and the C- and O-rich samples. 
The paper is organized as follows. We briefly recall the general scheme of our calibration strategy, and we describe the input data and AGB observations in Sect. 2. The adopted stellar models are presented in Sect. 3. We present the results of our LMC calibration in Sect. 4 and we discuss them in Sect. 5. Final remarks close the paper in Sect. 6.

\section{DATA AND METHODS}

As thoroughly described in Pastorelli et al. (2019) in the case of the SMC galaxy, our work relies on three fundamental components, now regarding the LMC galaxy:

(i) The spatially-resolved SFH derived for well-defined subregions of the sky across the LMC. Importantly, the SFH is derived from regions of the colour-magnitude diagram (CMD) in which the expected number of TP-AGB stars is negligible. This way we prevent the SFHs to be affected by uncertainties in the TP-AGB models themselves. Moreover, the same SFH-recovery process produces estimates of the distance and extinction of each subregion.

(ii) AGB catalogues that include an accurate identification of C- and O-rich type stars, mainly derived from a combination of Two Micron All-Sky Survey (2MASS) and Spitzer photometric data, and complemented by additional information, e.g. from spectroscopic surveys.

Regions of the LMC with available data for both the items above are modelled with the TRILEGAL stellar population synthesis code. This procedure makes use of the SFH, distances, and extinctions from item (i), and produces the theoretical counterparts of the catalogues from item (ii). Such simulations make use of our third basic component:

(iii) Extended grids of evolutionary models for TP-AGB stars, computed for different choices of the parameters describing to 3DU events and mass loss.

In the following, we briefly describe components (i) and (ii). The models from (iii) will be introduced one-by-one in Sects. 3 and 4, together with their comparison with the catalogues from (ii).

\subsection{Star Formation History}

We use the SFH derived from deep near-infrared data $(J$, $Y, K_{\mathrm{s}}$ filters) from the VISTA survey of the Magellanic Clouds (VMC; Cioni et al. 2011). We refer to Rubele et al. (2018, and references therein) for a complete description of the method, and its application to VMC data for the SMC. Briefly, the $\mathrm{SFH}$ is derived from two different CMDs, $K_{\mathrm{S}}$ vs. $J-K_{\mathrm{S}}$ and $K_{\mathrm{S}}$ vs. $Y-K_{\mathrm{S}}$, by finding the model that minimizes a data-model $\chi^{2}$-like statistic. The model CMDs are built with the TRILEGAL population synthesis code (the version by Marigo et al. 2017), using PARSEC v1.2S (Bressan et al. 2012) stellar evolutionary tracks. The method fits not only the stellar mass formed in several age bins, but also the age-metallicity relation (AMR), distance, and mean extinction for each analysed subregion. Throughout this work, 'SFH' refers to the combination of the star formation rate and metallicity as a function of age. The derivation of the SFH of the LMC will be fully described in a forthcoming paper.

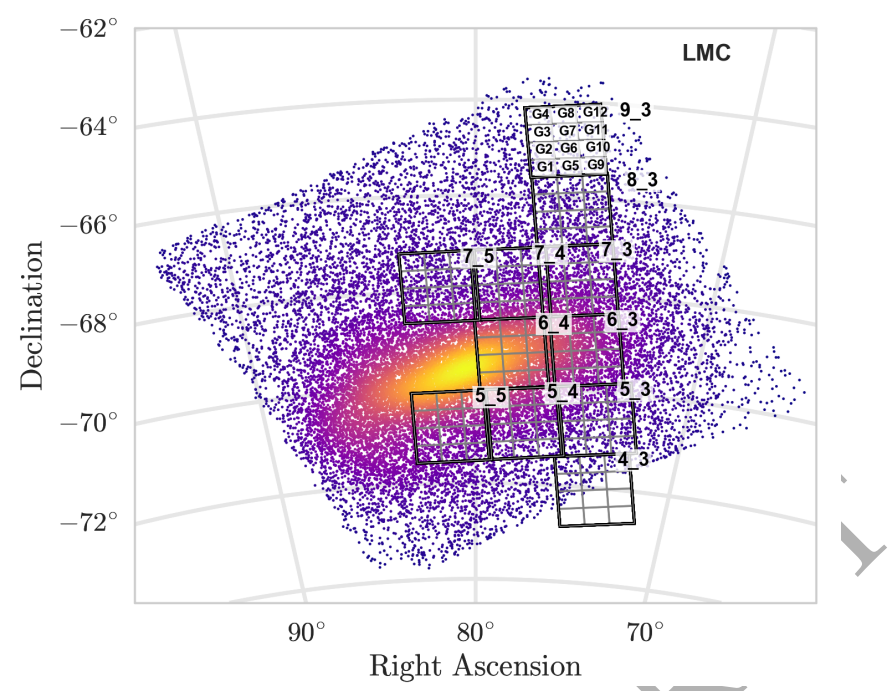

Figure 1. VMC tiles for which the SFH recovery is available. Each tile (in black) is subdivided into 12 subregions (in grey), as illustrated for the tile LMC 9_3. The background image shows the density map of the AGB stars classified by B11.

Table 1. Central sky coordinates of the VMC tiles and number of AGB stars identified by B11 for each tile.

\begin{tabular}{lccc}
\hline Tile & $\begin{array}{c}\text { R.A.J2000 } \\
(\mathrm{h}: \mathrm{m}: \mathrm{s})\end{array}$ & $\begin{array}{c}\text { Dec.J2000 } \\
(\mathrm{d}: \mathrm{m}: \mathrm{s})\end{array}$ & N. AGB \\
\hline LMC 4_3 & $04: 55: 19.5$ & $-72: 01: 53.4$ & 130 \\
LMC 5_3 & $04: 56: 52.5$ & $-70: 34: 25.7$ & 628 \\
LMC 5_4 & $05: 10: 41.5$ & $-70: 43: 05.9$ & 1070 \\
LMC 5_5 & $05: 24: 30.3$ & $-70: 48: 34.2$ & 1558 \\
LMC 6_3 & $05: 00: 42.2$ & $-69: 08: 54.2$ & 1634 \\
LMC 6_4 & $05: 12: 55.8$ & $-69: 16: 39.4$ & 2852 \\
LMC 7_3 & $05: 02: 55.2$ & $-67: 42: 14.8$ & 920 \\
LMC 7_4 & $05: 14: 06.4$ & $-67: 49: 21.7$ & 851 \\
LMC 7_5 & $05: 25: 58.4$ & $-67: 53: 42.0$ & 647 \\
LMC 8_3 & $05: 04: 55.0$ & $-66: 15: 29.9$ & 458 \\
LMC 9_3 & $05: 06: 40.6$ & $-64: 48: 40.3$ & 155 \\
\hline \multicolumn{5}{c}{ Total area } \\
Notes: & 25 deg ${ }^{2}$ & 10903 \\
\hline Excluded subregions: LMC 4_3 G1, G2, G5, G6, G9, G10, G11
\end{tabular}

Fig. 1 shows the VMC tiles for which the SFH is currently available in the LMC, superimposed on the density map of the AGB stars classified by B11. Table 1 summarizes their central coordinates, and the number of AGB stars in each tile. These tiles are about $1.5^{\circ} \times 1.0^{\circ}$ large $^{1}$, and their longer dimension runs almost along the North-South direction (Cioni et al. 2011). For the SFH analysis, each tile is divided into 12 subregions, labelled from G1 to G12 as indicated in Fig. 1.

In the case of the LMC, the observed photometry can be significantly affected by crowding, which in turn affects the SFH robustness. For this reason, we use the SFH solutions derived from the $K_{\mathrm{S}}$ vs. $J-K_{\mathrm{S}}$ and $K_{\mathrm{S}}$ vs. $Y-K_{\mathrm{S}}$ CMDs to simulate the VMC data of the LMC for each subregion to assess the quality of the solutions. For each subregion

\footnotetext{
1 The size of the tiles corresponds to the area where each pixel of the tile image is observed at least twice.
} 
we compute 10 TRILEGAL simulations and we compare the median number of simulated red giant branch (RGB) stars with the observed one in the $K_{\mathrm{S}}$ vs. $J-K_{\mathrm{S}} \mathrm{CMD}$, as shown in Fig. 2. The CMD region used to select RGB stars is such that 1) we avoid AGB contamination, 2) we exclude CMD regions that are likely to be severely affected by crowding errors and incompleteness. The results of these tests are shown in Fig. 3, plotted in terms of a fractional error in the RGB counts and standard deviations from the expected numbers.

From these figures, it is evident that some subregions (and even entire tiles) present large errors in their predicted RGB star counts. In some tiles (in particular the LMC 5_5, 6_4 and 7_5), the discrepancies are likely linked to the severe crowding conditions close to the LMC Bar, which has probably affected the $\mathrm{SFH}$ derivation in unexpected ways. It is beyond the scope of this paper to delve into the possible causes of these discrepancies. Moreover, there would be no easy solution to these problems given the large amount of computer time involved in performing the PSF photometry, and millions of artificial star tests over the VMC images, necessary to perform the SFH-recovery (see Rubele et al. 2018). Based on these tests, we culled our list of LMC subregions, keeping those for which the errors in RGB star counts are smaller than a given threshold. To this aim, we classify our subregions in four broad categories: 'Gold' are those in which RGB star counts are reproduced within $1 \sigma$, 'Silver' are between $1 \sigma$ and $3 \sigma$, and 'Bronze' are between $3 \sigma$ and $5 \sigma$, as illustrated in Fig. 3. Subregions with RGB star counts errors above $5 \sigma$ ('Rejected' regions) are not considered in the calibration. We notice that the mismatch between model and data RGB counts is generally smaller or similar to $\approx 10$ per cent for Gold and Silver subregions.

\subsection{Selected areas and their AGB numbers}

Table 2 shows the number of AGB stars that we can use in the calibration, depending on whether we choose to use 1) Gold, 2) Gold + Silver, or 3) Gold + Silver + Bronze subregions. We also distinguish between whether the $\mathrm{SFH}$ solution is obtained from the $K_{\mathrm{S}}$ vs. $J-K_{\mathrm{S}}$ CMD alone, or from both the $K_{\mathrm{S}}$ vs. $J-K_{\mathrm{S}}$ and the $K_{\mathrm{S}}$ vs. $Y-K_{\mathrm{S}}$ CMDs.

There is obviously a trade-off between adopting more inclusive criteria, and including regions with larger errors in their SFHs (as indicated by the mismatches in their RGB star counts). In this work, we consider the Gold+Silver VMCs subregions, for which the RGB star counts are reproduced within $3 \sigma$. Moreover, we decide to use the $\mathrm{SFH}$ solutions obtained from the $K_{\mathrm{S}}$ vs. $J-K_{\mathrm{S}} \mathrm{CMD}$ alone, so to maximize the number of AGB stars available, i.e. 4664 sources in 72 subregions. Despite the total number of AGB stars is lower than the number used in our previous work for the SMC (Pastorelli et al. 2019), it is large enough to reach a TP-AGB calibration of a similar quality, even with the present partial coverage of the LMC galaxy.

\subsection{Observations of AGB stars in the LMC}

The calibration performed in this work is based on the LMC AGB population classified by B11. They combined data from the 2MASS (Skrutskie et al. 2006) and SAGE-LMC surveys to study the evolved population of the LMC, and to give a
Table 2. Number of VMC subregions for which the simulated RGB star counts agree within $1 \sigma$ ('Gold'), $3 \sigma$ ('Gold+Silver'), and $5 \sigma$ ('Gold+Silver+Bronze') of the observed RGB star counts. The resulting number of AGB stars from the B11 catalogues are shown. The results are presented for the SFH solutions from the $J-K_{\mathrm{S}}$ and $J-K_{\mathrm{s}} ; Y-K_{\mathrm{S}}$ CMDs.

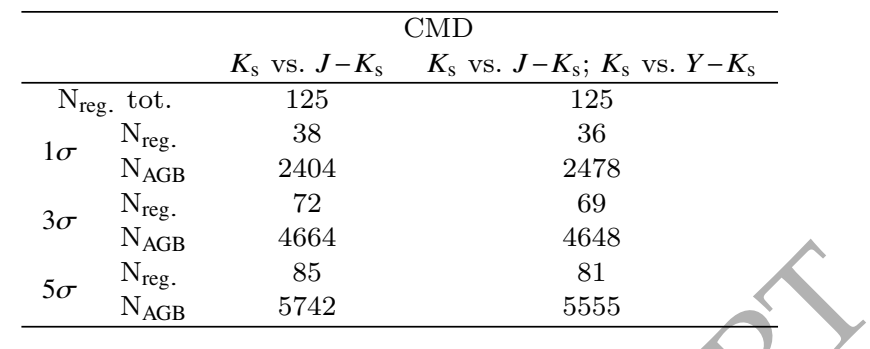

photometric classification of the AGB stars. The area covered by the SAGE-LMC survey is shown in Fig. 1.

The SAGE-LMC catalogue is a complete census of AGB stars in the LMC, including optically visible O- and C-rich stars above the tip of the RGB, as well as the most obscured dusty sources. The AGB stars are classified in O-rich (or O-AGB), C-rich (or C-AGB), extreme-AGB (X-AGB), and anomalous-AGB (a-AGB).

$\mathrm{O}$ - and C-rich sources are classified based on their position in the $K_{\mathrm{S}}$ vs. $J-K_{\mathrm{S}}$ CMD. The class of X-AGBs, first introduced by Blum et al. (2006), includes the very dusty stars, empirically selected on the basis of their $J-[3.6]$ and [3.6]-[8.0] colours. Most of them are C-rich stars, but a small number of O-rich is also present (van Loon et al. 2005).

The chemical type of sources classified as a-AGB cannot be photometrically inferred from the available combinations of 2MASS and Spitzer colours. However, for a subsample of them, Boyer et al. (2015b, hereafter B15) used optical spectrafrom Olsen et al. (2011) to determine their spectral type in both SMC and LMC. In the LMC, the percentage of aAGB stars that are O-rich is about 77 per cent, whereas in the SMC the percentage is about 50 per cent. The total number of a-AGB stars spectroscopically analysed by B15 in the LMC is 613 , leaving about 5800 a-AGB stars not classified as O- or C-rich. On the basis of the selection criteria adopted in Sect. 2.2, the number of a-AGBs considered here is 1076 .

In Paper I, we took into account the contribution of a-AGB stars by weighting the observed luminosity functions of the C-AGB and O-AGB using the fraction of aAGB sources spectroscopically classified as either C-rich or O-rich. Here, we apply a diagnostic tool proposed by Lebzelter et al. (2018) to assign the chemical type to the sample of a-AGBs. We refer to this diagram as Gaia-2MASS diagram, as it combines the Gaia Data Release 2 (DR2; Gaia Collaboration et al. 2018) and 2MASS Wesenheit functions ${ }^{2}$

To construct the Gaia-2MASS diagram for the a-AGB sample, we cross-match the SAGE-LMC catalogue with the Gaia DR2 data. For each source with both $\mathrm{J}$ and $K_{\mathrm{s}}$-band magnitudes from 2MASS, we obtain the Gaia counterpart

2 The expressions for the Gaia and 2MASS Wesenheit functions are

$W_{\mathrm{RP}, \mathrm{BP}}=G_{\mathrm{RP}}-1.3 \times\left(G_{\mathrm{BP}}-G_{\mathrm{RP}}\right)$ and $W_{\mathrm{J}, \mathrm{K}}=K_{\mathrm{s}}-0.686 \times\left(J-K_{\mathrm{s}}\right)$ respectively. 


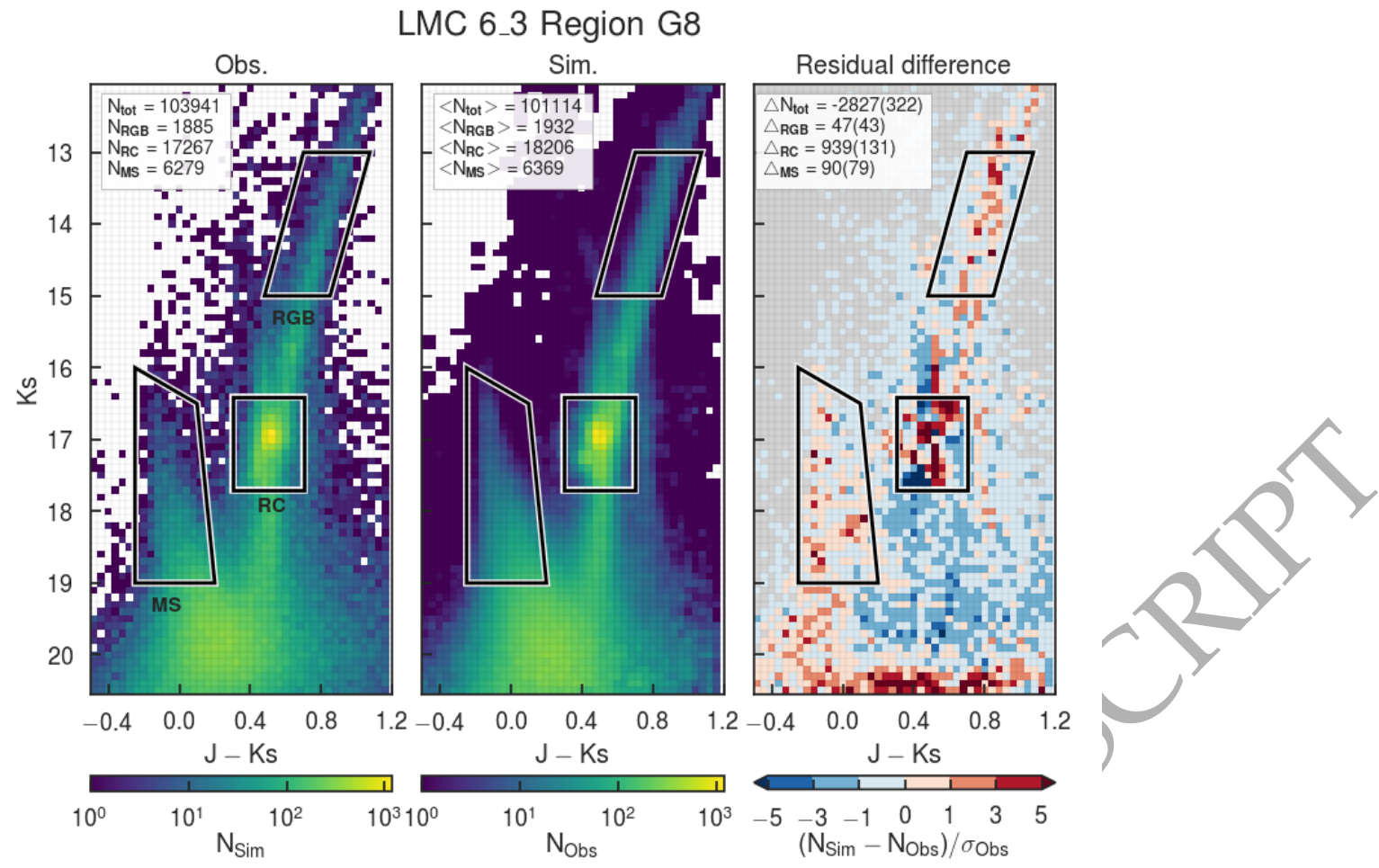

Figure 2. Comparison between the observed VMC data (left panel) and the simulated Hess diagram (middle panel) derived from the $K_{\mathrm{S}}$ vs. $\boldsymbol{J}-K_{\mathrm{S}}$ solution for the subregion G8 of tile LMC 6_3. The simulated diagram is the median of 10 TRILEGAL realizations. The right panel shows the difference between the model and the observed data, divided by the square root of the observed number counts $\left(\sigma_{\mathrm{Obs}}\right)$, so as to reveal the CMD regions with the most significant residuals. The boxes correspond to the RGB, Red Clump (RC) and upper Main Sequence (MS) regions. The total number of stars, and the number of stars in each box, are reported in the legend of each panel. In addition, the legend in the right panel indicates, in brackets, the $1 \sigma$ value of the observed number counts in the corresponding box.

using a search radius of $5^{\prime \prime}$. We further check our results using the pre-computed cross-match of Gaia DR2 with 2MASS ('2MASS BestNeighbour' table, Marrese et al. 2019). We find 1072 matches out of 1076 a-AGBs.

We first compare the classification from the Gaia2MASS diagram with the results of B15 for the a-AGBs with a spectroscopic classification (see left and middle panels of Fig. 4). The Gaia-2MASS method is in perfect agreement with the spectroscopic one for the sample of a-AGBs considered, with all the available sources correctly classified. Given these results, we classify the a-AGB stars for which no spectral information is available from B15 according to the position in the Gaia-2MASS diagram as shown in the right panel of Fig. 4.

We perform a further check on all the sources for which the classification is only based on the photometry from B11 and from the Gaia-2MASS diagram. We use the spectroscopic classification from Groenewegen \& Sloan (2018) based on Spitzer IRS spectra, the C-star catalogue by Kontizas et al. (2001), and the catalogue of MK spectral types compiled by Skiff (2014). By using a search radius of $2^{\prime \prime}$, we find a total of 807 counterparts. We correct the photometric classification of $3 \mathrm{C}$-rich and 47 O-rich stars that are spectroscopically classified as $\mathrm{M}$ - and C-type, respectively.

Table 3 lists the final number counts of C-, O-, X-, and a-AGB stars used in this work. The contribution of the remaining 4 a-AGBs with no counterpart in Gaia DR2 is taken
Table 3. Number counts of C-, O-, X-, and a-AGB stars. The final number counts used in this work include the contribution of a-AGB stars from B15 without Gaia DR2 counterparts.

\begin{tabular}{lcc}
\hline Population & N. star & N. star (this work) \\
\hline C-AGB & 1453 & 1454 \\
O-AGB & 2931 & 2934 \\
X-AGB & 276 & 276 \\
a-AGB & 4 & 0 \\
\hline
\end{tabular}

into account by weighting the LFs as in Paper I, using the fraction of O- and C-rich a-AGBs from B15.

\subsection{TRILEGAL simulations and model selection criteria}

Our calibration strategy, including the details of the AGB selection criteria adopted in the models are extensively described in Paper I. We briefly summarize them here.

We simulate the photometry of each LMC subregion selected in Sect. 2.2 with the TRILEGAL code, and we merge all the synthetic catalogues to be compared with the observed AGB catalogue. This latter only includes the sources located in the same sky area as the VMC subregion.

Each subregion is modelled according to its SFR, AMR, distance, and reddening derived from the SFH recovery. We adopt the Kroupa (2001) initial mass function for single stars, and we simulate non-interacting binaries using a bi- 

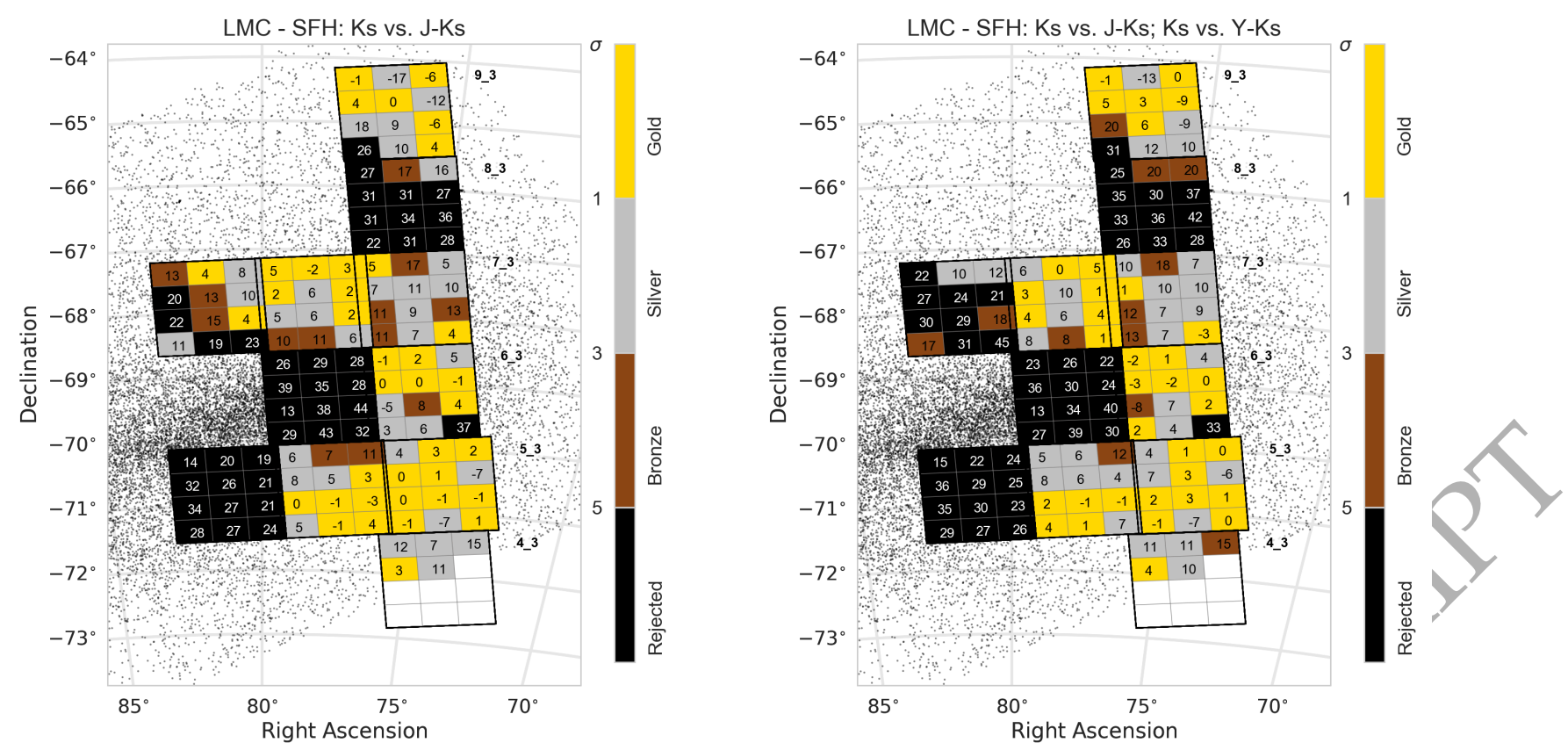

Figure 3. Maps of the predicted vs. observed RGB star counts across the LMC area considered in this work. The two maps show the results obtained with the input SFH solutions coming from the $K_{\mathrm{s}}$ vs. $J-K_{\mathrm{s}}$ CMDs (left panel), and from the combined use of $K_{\mathrm{s}}$ vs. $J-K_{\mathrm{S}}$ and $K_{\mathrm{S}}$ versus $Y-K_{\mathrm{S}}$ CMDs (right panel). For each subregion we report the percentage of the difference between the number of simulated and observed RGB stars, i.e. $100 \times\left(N_{\mathrm{sim}}^{\mathrm{RGB}}-N_{\mathrm{obs}}^{\mathrm{RGB}}\right) / N_{\mathrm{obs}}^{\mathrm{RGB}}$. The color-code of each subregion is the following: 'Gold' for RGB star counts within $1 \sigma$, 'Silver' for star counts between 1 and $3 \sigma$, 'Bronze' for star counts between 3 and $5 \sigma$. Subregions with differences in RGB star counts above $5 \sigma$ are not considered in the calibration.

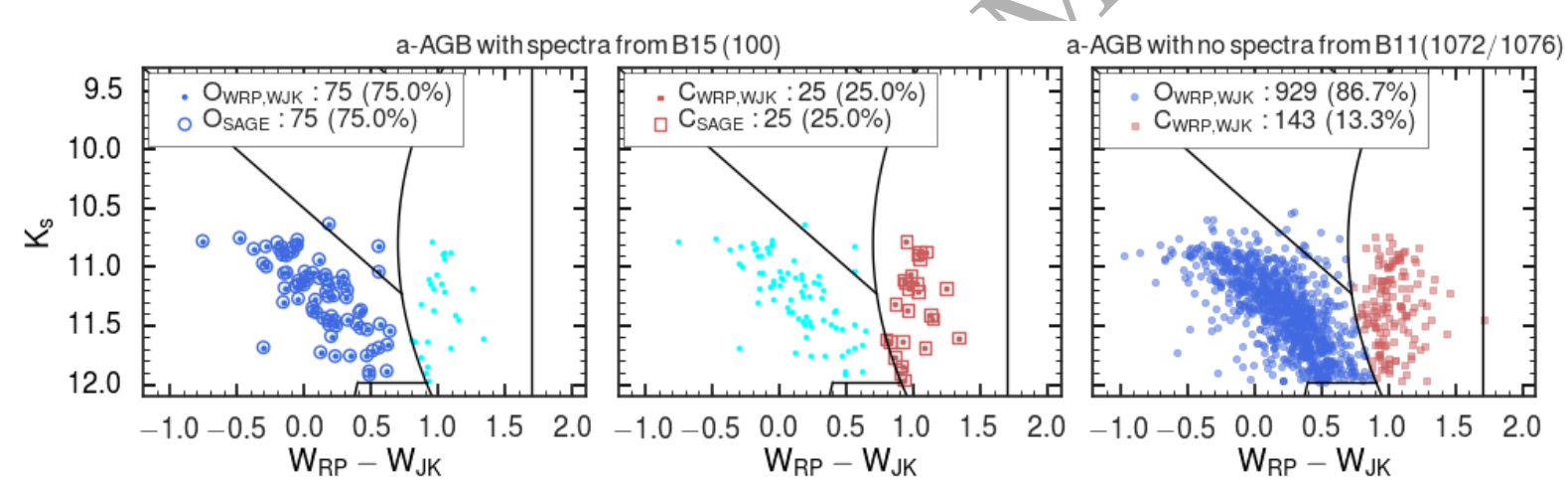

Figure 4. Location of the a-AGB stars in the Gaia-2MASS diagram. The black lines correspond to the regions described by Lebzelter et al. (2018) with the bending curve dividing O-rich (left) and C-rich (right) stars. The sample of a-AGBs with spectra from B15 are shown as cyan dots in the left and middle panels. Left panel: a-AGB stars classified as O-rich by B15 are marked with blue open circles $\left(\mathrm{O}_{\mathrm{SAGE}}\right)$, and those classified as O-rich in the Gaia-2MASS diagram $\left(\mathrm{O}_{\mathrm{WRP}, \mathrm{WJK}}\right)$ as blue dots. Middle panel: a-AGB stars classified as C-rich by B15 are shown as red open squares $\left(\mathrm{C}_{\mathrm{SAGE}}\right)$, and those classified as C-rich in the Gaia-2MASS diagram ( $\left.\mathrm{C}_{\mathrm{WRP}, \mathrm{WJK}}\right)$ as red squares. Right panel: a-AGB stars, for which spectroscopic information is not available, classified using the Gaia-2MASS diagram. The legend in each panel/shows the number of classified sources and their percentage with respect to the total. Only the sources selected for this work are shown. The number of a-AGB stars with spectra from B15 is 100, all of them have a Gaia DR2 counterpart. The number of a-AGBs, from B11 with no spectra is 1076, and we find a Gaia DR2 match for 1072 of them.

nary fraction of 30 per cent along with a uniform mass distribution of mass ratios between the secondary and the primary components in the range $0.7-1$. The photometric errors are taken into account in TRILEGAL following the distribution of errors as a function of magnitude reported in the observed catalogue.

The Milky Way foreground and incompleteness of the data are not simulated, simply because these effects are less of a problem in the CMD region occupied by AGB stars. The 2MASS catalogue is complete down to $K_{\mathrm{S}}=14.3 \mathrm{mag}$ (Skrutskie et al. 2006), that is $\approx 2$ mag fainter than the tip of the RGB in the LMC. Furthermore, AGB stars are identified by combining both near- and mid-infrared photometry (B11). This ensures that the most obscured dusty stars are not missed from the observed catalogues. Melbourne \& Boyer (2013) estimated the foreground con- 
tamination for the AGB population in the LMC to be below 1 per cent.

To select AGB stars and the three classes of C-, O-, and $\mathrm{X}$-AGB in the synthetic catalogues we use a combination of theoretical parameters and photometric criteria. The $\mathrm{C} / \mathrm{O}$ ratio is used to select $\mathrm{C}$ - and $\mathrm{O}$-rich stars. The class of $\mathrm{O}-$ rich stars contains both early-AGB and TP-AGB stars and we use the same photometric criteria as in B11. X-AGB stars are selected using photometric criteria alone. We refer to Section 2.5 and Appendix A.2 in Paper I for a complete description.

We produce 10 TRILEGAL realizations for each subregion, and we calculate the $\chi^{2}$ of the median LF, $\chi_{\mathrm{LF}}^{2}$, with respect to the data. A satisfactory agreement between data and model is achieved when we obtain the lowest $\chi_{\mathrm{LF}}^{2}$ values for the entire sample, and for the three classes of AGB stars.

In Paper I, we identified a systematic shift in the $J-K_{\mathrm{S}}$ colour of the synthetic RSG and O-rich stars in the SMC. The most likely explanation for this discrepancy is a temperature offset. A mismatch between the predicted and observed colours may hamper our comparison with the observed Orich stars, specifically the RSG and O-rich separation, which is based on photometric criteria. Paper I corrected the synthetic photometry of RSGs and O-rich AGBs to match the observed RSG colours. In this work we avoid making such corrections as in the case of the LMC we do not not find a significant shift in the RSG colour. However, the O-rich AGBs show a similar shift to redder colours comparable to the SMC case, i.e. $\Delta J-K_{\mathrm{S}} \approx 0.05-0.1$ between $K_{\mathrm{S}} \approx 12.7-11.8 \mathrm{mag}$. While an assessment of this discrepancy will be the subject of a future work, we emphasize that this only affects the number of O-rich stars by about 5 per cent.

\section{STELLAR EVOLUTIONARY MODELS}

We adopt the PARSEC database (Bressan et al. 2012) of stellar evolutionary models to cover all phases from the premain sequence up to carbon ignition in massive stars, or up to the occurrence of the first thermal pulse in low- and intermediate-mass stars. For these latter stars the TP-AGB phase is then computed with the COLIBRI code (Marigo et al. 2013). In this section, we recall the main features of our TP-AGB models, and we refer to Marigo et al. (2013) and Pastorelli et al. (2019) for a detailed description.

The COLIBRI calculations start from the stellar configuration given by the PARSEC models at the beginning of TP-AGB phase. The equation of state and the gas opacities are computed on-the-fly with the ESOPUS code (Marigo \& Aringer 2009), so as to consistently follow the variations in the chemical composition caused by mixing events and nucleosynthesis.

Mass loss by stellar winds during the AGB phase is described with a two-regime scheme, first introduced in Girardi et al. (2010):

(i) Pre-dust mass loss $\left(\dot{M}_{\text {pre-dust }}\right)$. It applies as long as the conditions, mainly at lower luminosities and higher effective temperatures, prevent the formation of dust grains and the development of a dust-driven wind. In this work we adopt the formalism presented by Cranmer \& Saar (2011), which relies on the action of magnetic fields in the extended and cool chromospheres of red giants. In our models the pre-dust mass loss typically takes place during the Early-AGB phase.

(ii) Dust-driven mass loss ( $\left.\dot{M}_{\text {dust }}\right)$. When AGB stars evolve to higher luminosities and large-amplitude pulsation develops, powerful stellar winds may be triggered through radiation pressure on dust grains which form in the extended and shocked atmospheres (Höfner \& Olofsson 2018). Here we adopt different mass-loss descriptions depending on the surface $\mathrm{C} / \mathrm{O}$ ratio. As long as a star has $\mathrm{C} / \mathrm{O}<1$, we use the Bloecker (1995, hereafter BL95) formula, with an efficiency parameter $\eta_{\text {dust }}=0.03$. When the star attains $\mathrm{C} / \mathrm{O}>1$, as a consequence of the 3DU, we adopt the results of dynamical atmospheres models for carbon stars (hereafter CDYN) recently developed by Mattsson et al. (2010), Eriksson et al. (2014), and Bladh et al. (2019).

As detailed in Marigo et al. (2013), the 3DU is modelled with a parametrized description that relies on three main characteristics:

(i) Onset and quenching of the $3 D U$. These are determined by a temperature criterion, $T_{\mathrm{b}}^{\mathrm{dred}}$, which is the minimum temperature that must be reached at the base of the convective envelope at the stage of post-flash luminosity maximum for a mixing event to occur.

(ii) Efficiency of the $3 D U$. It is described by the standard parameter $\lambda=\Delta M_{\text {dred }} / \Delta M_{\mathrm{c}}$, the fractional increment of the core mass during an inter-pulse period that is dredged up in the subsequent thermal pulse. In this work we adopt the new parametrization of $\lambda$ introduced by Pastorelli et al. (2019). This scheme is designed to be: a) qualitatively consistent with full TP-AGB model calculations, and b) to contain suitable free parameters to perform a physically-sound calibration based on the observed C-rich star LFs. The free parameters are: i) $\lambda_{\max }^{*}$, the maximum efficiency of the $3 \mathrm{DU}$ among all TP-AGB models; ii) $\widetilde{M}_{\mathrm{c}}$, the value of the core mass for which $\lambda_{\max }^{*}$ is attained; and iii) $M_{\mathrm{c}, \lambda=0}$, the value of the core mass above which the $3 \mathrm{DU}$ is no more active. This latter parameter is introduced to allow for the possibility that at larger core masses the average efficiency of the 3DU may decline, as indicated by some existing TP-AGB models (Ventura \& D'Antona 2009; Cristallo et al. 2015).

(iii) Chemical composition of the intershell. Here we adopt the standard case described in Marigo et al. (2013), where no overshoot is assumed at the convective boundaries, and the typical abundances of helium, carbon and oxygen are (in mass fraction): ${ }^{4} \mathrm{He} /{ }^{12} \mathrm{C} /{ }^{16} \mathrm{O} \approx(0.70-0.75) /(0.25-0.20) /(0.005-0.01)$.

During the calibration cycle, each time a new set of TP-AGB tracks is computed for a new combination of parameters, the next step is the generation of a corresponding set of PARSEC+COLIBRI stellar isochrones by means of the TRILEGAL code, as detailed in Marigo et al. (2017). We recall that TRILEGAL includes specific TP-AGB physical processes, such as the luminosity and temperature variations during the thermal pulse, the variations in the surface chemical compositions and spectral type, as well as the reprocessing of radiation by circumstellar dust. The photometry is calculated using extensive tables of bolometric corrections based on the spectral libraries by Aringer et al. (2009) for Crich stars, and Castelli \& Kurucz (2003) and Aringer et al. (2016) for O-rich stars. 
The synthetic photometry includes the effect of the circumstellar dust in mass-losing stars, as fully described by Marigo et al. (2008). Briefly, this approach is based on radiative transfer calculations across dusty envelopes (Groenewegen 2006; Bressan et al. 1998), coupled with the scaling formalism first introduced by Elitzur \& Ivezić (2001) and a few key relations from the dust-growth model by Ferrarotti \& Gail (2006). In Pastorelli et al. (2019) we made a few modifications to the dust treatment to improve the consistency of our simulations. We revised the abundance of some elements to follow the scaled-solar pattern of Caffau et al. (2011), as in the evolutionary tracks, and we replaced the fitting relations to compute the condensation degree of carbon dust by Ferrarotti \& Gail (2006) with the results of dynamical atmosphere models by Eriksson et al. (2014), which are also used to predict the mass-loss rates of C-rich stars during the dust-driven regime. For the present work we adopt tables of dust bolometric corrections based on spectra computed with the following dust mixtures: amorphous carbon (85 per cent) and $\mathrm{SiC}$ (15 per cent) for C-rich stars, and silicates for O-rich stars (Groenewegen 2006).

\section{RESULTS}

In this Section we present the results of our TP-AGB calibration for the LMC galaxy. We first describe our starting population synthesis model, and its performance compared to the observed LFs (see Sect. 4.1). Then, we present the best-fitting model we find by acting on the 3DU parameters (Sect. 4.2). Finally, in Section 4.3, we discuss the effect of newly available line lists for modelling the spectra of C-rich stars, and their impact on the TP-AGB model calibration.

\subsection{Starting model}

In Pastorelli et al. (2019) we identified two best-fitting models (the sets S_07 and S_35) which reproduce the SMC infrared LFs in the 2MASS and Spitzer filters, and the star counts for each class of AGB stars. Both models perform comparably well in recovering the observed LFs and CMDs, but with some preference towards the set S_35 as it yields final masses for the white dwarfs (WDs) which are closer to the semi-empirical initial-final mass relation (IFMR; El-Badry et al. 2018; Cummíngs et al. 2018).

We start by simulating the LMC photometry using COLIBRI TP-AGB evolutionary tracks with the S_35 input prescriptions, summarized in Table 4 . We show the performance of this set in Fig. 5. We note that while the number counts of the observed O-rich AGB stars are reasonably well reproduced, the faint end of the simulated LFs shows an excess which is compensated by a deficit at brighter magnitudes, i.e. $K_{\mathrm{s}} \approx 11.5-10.8 \mathrm{mag}$, Moreover, the most evident discrepancy is the overestimation of C-rich stars, especially at faint $K_{\mathrm{s}}$ magnitudes, by roughly 40 per cent. Similarly, a sizeable excess is found in the simulated X-AGB stars.

In this respect, we recall that the set $\mathrm{S} \_35$ was calibrated to reproduce the photometry of the SMC which, on average, has a lower metallicity than the LMC. In fact, the mean metallicity of SMC C-rich stars is $Z_{\mathrm{i}} \approx 0.004$, whereas the bulk of LMC C-rich stars has $Z_{\mathrm{i}} \approx 0.008$ (see Fig. 6). It follows that the excess of simulated C-rich and X-AGB stars
Table 4. Third dredge-up parameters of the TP-AGB sets presented in this work.

\begin{tabular}{cccc}
\hline \multirow{2}{*}{ SET } & \multicolumn{3}{c}{ Third dredge-up } \\
& $\lambda_{\max }^{*}$ & $\widetilde{\mathrm{M}}_{\mathrm{c}}\left[\mathrm{M}_{\odot}\right]$ & $\mathrm{M}_{\mathrm{c}, \lambda=0}\left[\mathrm{M}_{\odot}\right]$ \\
\hline S_35 & 0.7 & 0.60 & 1.00 \\
$\mathrm{~S} \_36$ & 0.7 & 0.70 & 1.00 \\
S_37 & 0.5 & 0.70 & 1.00 \\
\hline
\end{tabular}

in the LMC may be linked to the different metallicities that characterize the two galaxies. Hence, the need to check, and possibly revise, the starting TP-AGB calibration at higher metallicities.

In this work, we adopt the evolutionary tracks of the set $\mathrm{S} \_35$, already calibrated in the SMC, for the metallicity range $Z_{\mathrm{i}}=0.0005-0.006$. In addition, for higher metallicities, $Z_{\mathrm{i}}=0.008,0.01,0.014,0.017$, and 0.02 , we compute around 70 tracks with initial masses in the range between 0.5 and 5 $6 \mathrm{M}_{\odot}$, for each new combination of input parameters. This choice is also motivated by the fact that the predicted initial metallicity distributions of all classes of AGB/stars show a pronounced peak at $Z_{\mathrm{i}} \approx 0.008$, as shown in Fig. 6 . We emphasize that the metallicity distributions are based on the AMR from the SFH recovery. As such, the present calibration only probes metallicities as high as $Z_{\mathrm{i}} \approx 0.012$. We note that in our TRILEGAL simulations we use a set of COLIBRI tracks covering the entire metallicity range expected for the LMC population.

\subsection{Characterizing the 3DU in the LMC}

As a first attempt to reduce the number of low-mass faint C-rich stars, we compute the COLIBRI set S_36 in which we keep the same mass-loss prescription as in S_35, whereas we increase the $3 \mathrm{DU}$ parameter $\widetilde{\mathrm{M}}_{\mathrm{c}}$ from 0.6 to $0.7 \mathrm{M}_{\odot}$. To better appreciate the global effect, in Fig. 7 we show the efficiency of the $3 \mathrm{DU}$ as a function of the core mass $M_{C}$ for a few TP-AGB evolutionary models with $M_{\mathrm{i}} \geq 1.4 \mathrm{M}_{\odot}$ (top panels), and the corresponding simulated C-rich star LFs (bottom panels). In each panel $\widetilde{\mathrm{M}}_{\mathrm{c}}$ corresponds to the value of the core mass for which $\lambda$ attains the maximum value, $\lambda_{\max }^{*}$. The main effect of increasing this parameter is to delay the onset of the 3DU at larger core masses (in addition to the temperature criterion) in all TP-AGB models; in particular the occurrence of the mixing events is even prevented in stars with $M_{\mathrm{i}}<1.65 \mathrm{M}_{\odot}$ at $Z_{\mathrm{i}}=0.008$. This depopulates the faint wing of the C-rich LF, leading to a better agreement of the models with the observed data.

At the same time, increasing $\widetilde{\mathbf{M}}_{\mathrm{c}}$ shifts the maximum $3 \mathrm{DU}$ efficiency to stars of larger mass, from $M_{\mathrm{i}} \approx 2.4 \mathrm{M}_{\odot}$ in set $\mathrm{S} \_35$ to $M_{\mathrm{i}} \approx 3.0 \mathrm{M}_{\odot}$ in set $\mathrm{S} \_36$. The reduction in the number of C-rich stars is significant, and the simulated C-rich LF agrees better with the observed one.

The set S_36 improves in the simulated O-rich LF as well (see Fig. 8). In particular, it reduces the deficit of Orich stars in the bright wing of the $\mathrm{LF}\left(K_{\mathrm{S}} \lesssim 11.4 \mathrm{mag}\right)$. This is the consequence of delaying the onset of the $3 \mathrm{DU}$ in intermediate mass-stars $\left(M_{\mathrm{i}} \approx 2-3 \mathrm{M}_{\odot}\right)$, so that the O-rich stages extend over brighter luminosity bins.

Though the improvement obtained with the set S_36 is already appreciable, the calibration can be further refined to fill the deficit of C-rich stars in the bright-end of the sim- 


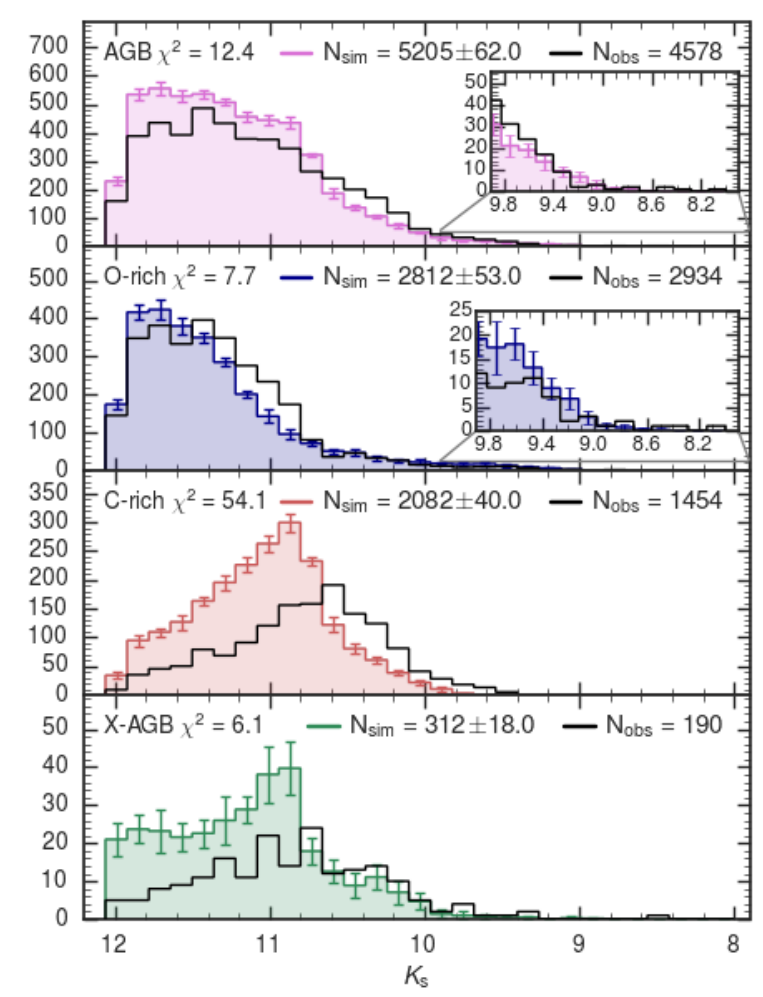

Figure 5. Simulated mean $K_{\mathrm{s}}$-band LFs based on S_35 models (filled histograms in colour), compared to observed distributions (dark-line histograms), for the entire sample (top panel) and for the three main classes of TP-AGB stars (other panels). The error bars cover 1- $\sigma$ uncertainties resulting from 10 TRILEGAL realizations. Each panel reports the numbers of observed and synthetic stars, as well as the $\chi^{2}$ specific to each simulated LF.

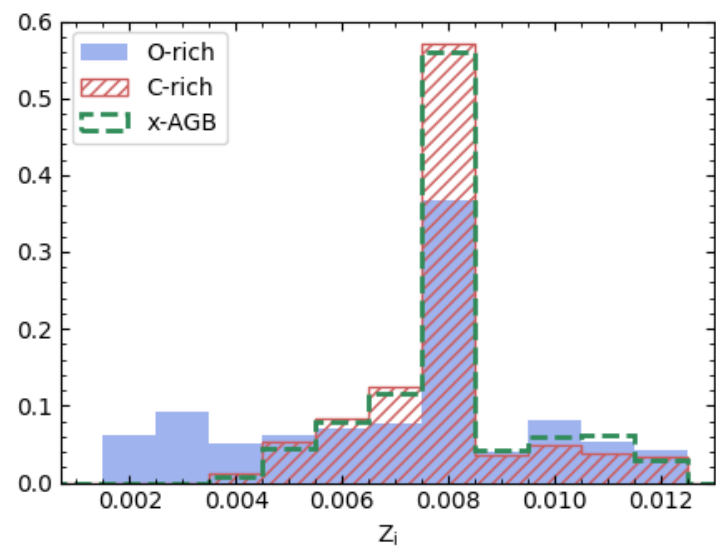

Figure 6. Initial metallicity distributions of O-, C-, and X-AGB stars predicted by S_35. The AMR is derived from the SFH recovery, and it is an input of our simulations.

ulated LF, by stretching the distribution to slightly brighter magnitudes, and to reduce the excess of C-rich and X-AGB stars fainter than $K_{\mathrm{S}} \approx 10.7 \mathrm{mag}$. Following the analysis carried out by Pastorelli et al. (2019), the natural step is to decrease the $\lambda_{\max }^{*}$ parameter. We compute the set S_37, in which $\lambda_{\max }^{*}$ is lowered from 0.7 to 0.5 . The net effect is a moderate reduction of $\lambda$ in all stellar models (see top right panel of Fig. 7), with the consequence that the transition to $\mathrm{C} / \mathrm{O}>1$, and the subsequent carbon-rich phases, take place at somewhat brighter magnitudes, resulting in a small shift of both C-rich and X-AGB distributions (bottom right panels of Figs. 7 and 9). The improvement obtained moving from S_36 to S_37 is quantitatively measured by comparing the $\chi_{\mathrm{LF}}^{2}$ of the simulated distributions, which decreases from 12.9 to 4.6 for the C-rich stars.

With the set S_37 we reach a satisfactory agreement with the observations. We obtain the lowest $\chi_{\mathrm{LF}}^{2}$ values for the entire AGB sample, and for the classes of O-, C- and $\mathrm{X}-\mathrm{AGB}$, at the same time. The total AGB number counts are matched within 10 per cent, while the predicted C-rich number counts are within 5 per cent. The residual excess of simulated O-rich stars, $\approx 15$ percent, can be reduced to $\approx 10$ percent by taking into account the mismatch in the predicted $J-K_{\mathrm{S}}$ colours, as discussed in Sect. 2.4. Despite the significant improvement in both the number counts and the shape of the X-AGB LF with respect to the starting model, a residual excess of simulated stars is present at brighter magnitudes, i.e. $K_{\mathrm{S}} \lesssim 11 \mathrm{mag}$. In this respect, we emphasize that our calibration is focused on reproducing the bulk of O- and C-rich AGBs, and it is not affected by the low numbers of X-AGB sources. Furthermore the X-AGB stars are photometrically selected, and their predicted magnitudes are largely dependent on the adopted circumstellar dust prescriptions.

In summary, our analysis leads us to conclude that the 3DU in TP-AGB stars of the LMC should be somewhat less efficient than in TP-AGB stars of the SMC. This result is consistent with the qualitative trends predicted by full TP-AGB models (e.g. Karakas et al. 2002; Ventura \& D'Antona 2009; Cristallo et al. 2011), and earlier calibration studies (e.g. Marigo \& Girardi 2007).

\subsection{Effects of new opacity data in carbon star spectra}

In recent years, new molecular linelists have become available for some of the species important to model carbon star spectra. Compared to the original grid of Aringer et al. (2009) the inclusion of these data in the calculation of atmospheric models and observable properties causes significant changes in the pressure-temperature structures, synthetic spectra and photometric colours. The largest effects are due to new linelists for $\mathrm{C}_{2}$ (Yurchenko et al. 2018) and $\mathrm{C}_{2} \mathrm{H}_{2}$ (Chubb et al. 2020), computed as part of the ExoMol project (Tennyson et al. 2016). Updated opacities were also published for HCN (Barber et al. 2014), $\mathrm{CH}$ (Masseron et al. 2014) and the lower levels of $\mathrm{CN}$ (Brooke et al. 2014; Sneden et al. 2014). However, the latter will only give rise to small changes in the overall energy distribution. The most important differences concerning the $J-K_{\mathrm{S}}$ indices of carbon stars are caused by the new $\mathrm{C}_{2} \mathrm{H}_{2}$ data, which produce a much lower opacity of this species in the range between 1 and $2 \mu \mathrm{m}$ resulting in considerably redder colours.

Fig. 10 compares the predicted $J-K_{\mathrm{S}}$ colours as a function of effective temperature between the previous sets of COMARCS models from Aringer et al. (2009, and including updates from Aringer et al. 2016) and the new set of mod- 

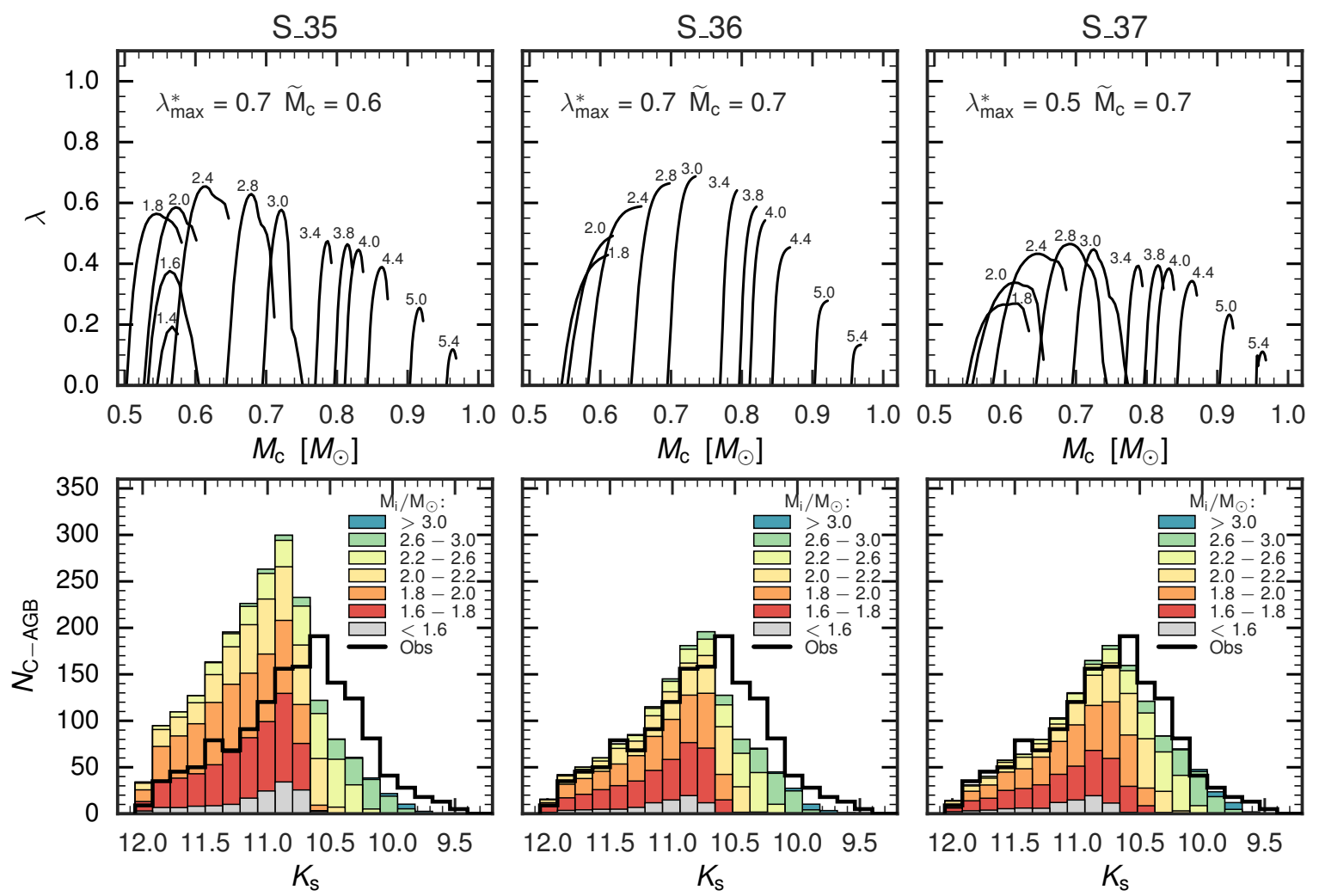

Figure 7. Top rows of each panel: efficiency of the 3DU $(\lambda)$ as a function of the core mass $M_{c}$ of a few selected evolutionary tracks with $Z_{\mathrm{i}}=0.008$ and initial mass as labelled in the figure. Bottom rows of each panel: observed (black histograms) and simulated C-rich LFs as derived from the corresponding above sets of models. The synthetic LFs are shown as stacked histograms to highlight the contribution of each initial mass bin to the LF as indicated in the legend.

els that include the new opacity data for C-rich stars. In this comparison, models are limited to a single value of surface gravity $(\log g=0)$, and refer to dust-free stars. The $J-K_{\mathrm{S}}$ colours obtained with the two opacity sets are the same for relatively high effective temperatures. However, for $T_{\text {eff }}$ values below $3200 \mathrm{~K}$, the $J-K_{\mathrm{S}}$ colours predicted by the new models become about 0.2 mag redder than those from Aringer et al. (2009).

The net effect of the new opacities on populations of AGB stars will depend on the distribution of $T_{\text {eff }}, \log g$, carbon excess, and dust properties of the model AGB stars. Unfortunately, the grid of new COMARCS models is not yet complete enough to be implemented straight away in TRILEGAL, or to model all these dependencies in a consistent way. However, the computed grid is large enough to assess the differences in the predicted magnitudes between the two versions of the same/simulation. Therefore, we derive a relation that yields the corrections to be applied to the magnitudes given by the standard set of spectra in order to recover the results obtained with the new opacity data. This is based on COMARCS models at solar metallicity, but the effect for lower metallicity C-rich stars is expected to go in the same direction. The correction contains a dependence on both effective temperature and carbon excess. We apply it to each C-rich star of the S_37 simulation. The main effect of the new opacity data is to predict brighter $K_{\mathrm{S}}$ magnitude for stars with effective temperatures below $3200 \mathrm{~K}$. Adopting this correc- tion leads to a better fit to the observed bright wing, as proved by the lower value of the $\chi^{2}$ which decreases from 4.6 down to 2.1 for the set $\mathrm{S} \_37$. The most evident improvement is found in the predicted near-IR colours. In Fig. 11 we show the observed and simulated $K_{\mathrm{S}}$ vs. $J-K_{\mathrm{S}}$ Hess diagrams for the C-rich population. For the Aringer et al. (2009) models, the $J-K_{\mathrm{S}}$ colours reach a maximum value of $\approx 1.6 \mathrm{mag}$, with the bulk of C-rich stars aligning along an almost vertical structure at brighter magnitudes. Conversely, the bulk of the observed C-rich population shows redder $J-K_{\mathrm{S}}$ colours at brighter $K_{\mathrm{S}}$ magnitude. The observed behaviour is now better reproduced if we adopt the correction derived from the new opacity data. The slope of the simulated C-rich sequence shows a bending towards redder $J-K_{\mathrm{S}}$ colours similar to the observations. The improvement in the $J-K_{\mathrm{S}}$ vs. $K_{\mathrm{S}}$ diagram can also be appreciated by considering, for each cell of the Hess diagram, the difference between the number of simulated and observed number counts, relative to $1 \sigma$ of the predicted distribution (Fig. 11). The value of the $\chi^{2}$ is reduced from 6.35 down to 3.42 as we move from the old to the new simulations.

To test the impact of the new opacity on our previous TP-AGB calibration (Pastorelli et al. 2019) we perform the same kind of test on the SMC and apply the correction to the simulations calculated with the best-fitting set S_35. We find no significant changes in the $K_{\mathrm{s}}$-band LFs, nor in the $K_{\mathrm{S}}$ vs. $J-K_{\mathrm{S}}$ CMDs. The reason is that C-rich stars 


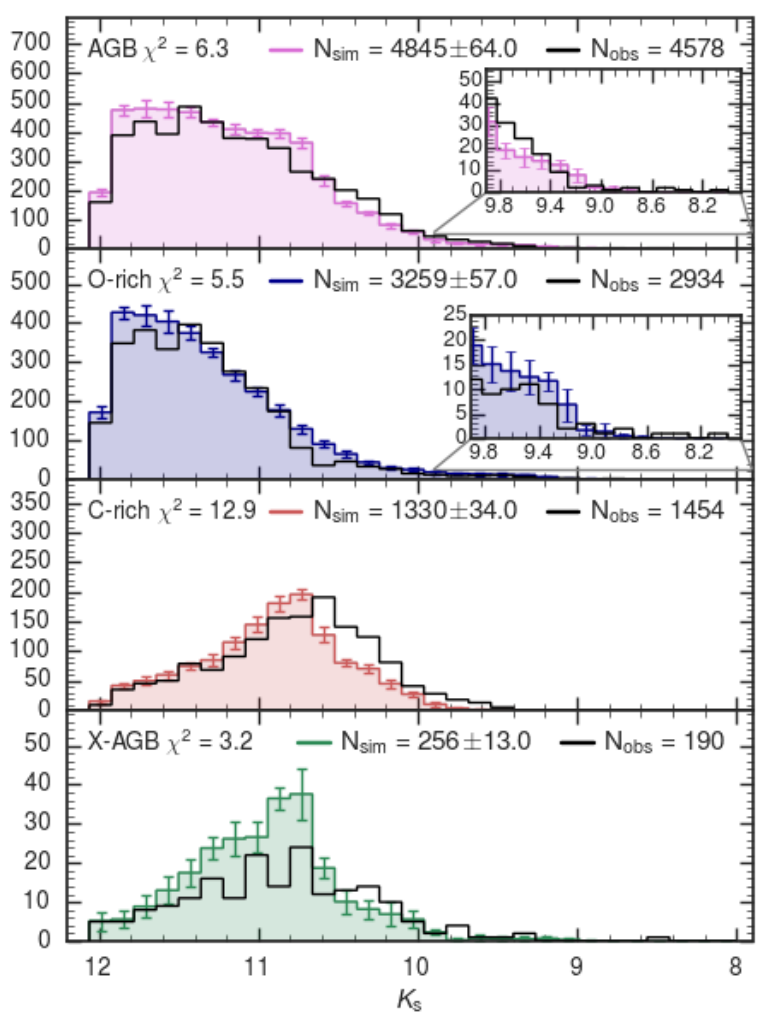

Figure 8. Same as Fig. 5, but for S_36 in which we increase the $3 \mathrm{DU}$ parameter $\widetilde{\mathrm{M}}_{\mathrm{c}}$ from 0.6 to $0.7 \mathrm{M}_{\odot}$.

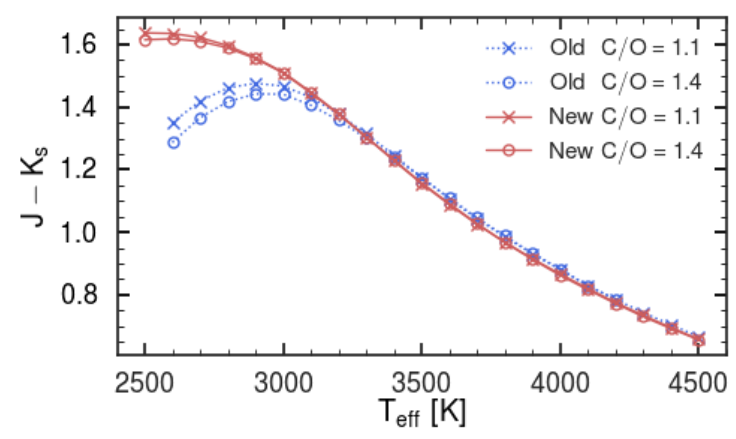

Figure 10. Predicted $J-K_{\mathrm{s}}$ colour as a function of effective temperature calculated from COMARCS models with a surface gravity of $\log \left(\mathrm{g}\left[\mathrm{cm} / \mathrm{s}^{2}\right]\right)=0.0$, a stellar máss of $M=1 \mathrm{M}_{\odot}$ and solar abundances except for carbon. Two $\mathrm{C} / \mathrm{O}$ ratios (1.1 - crosses, 1.4 circles) are shown. The results are based on the standard grid by Aringer et al. (2016, old, blue dotted lines) and on computations with the new opacities for $\mathrm{C}_{2} \mathrm{H}_{2}$ and $\mathrm{C}_{2}$ (new, red solid lines).

in the SMC have, on average, higher effective temperatures compared to the LMC, and this characteristic makes the correction smaller for the SMC, as shown in Fig. 10.

\subsection{Observed and best-fitting $K_{\mathrm{S}}$ vs. $J-K_{\mathrm{S}}$ CMDs}

We show the comparison between the observed and the simulated $K_{\mathrm{S}}$ vs. $J-K_{\mathrm{S}}$ CMDs from the best-fitting model S_37 in Fig. 12. In the observed CMD, the stars are colour-coded

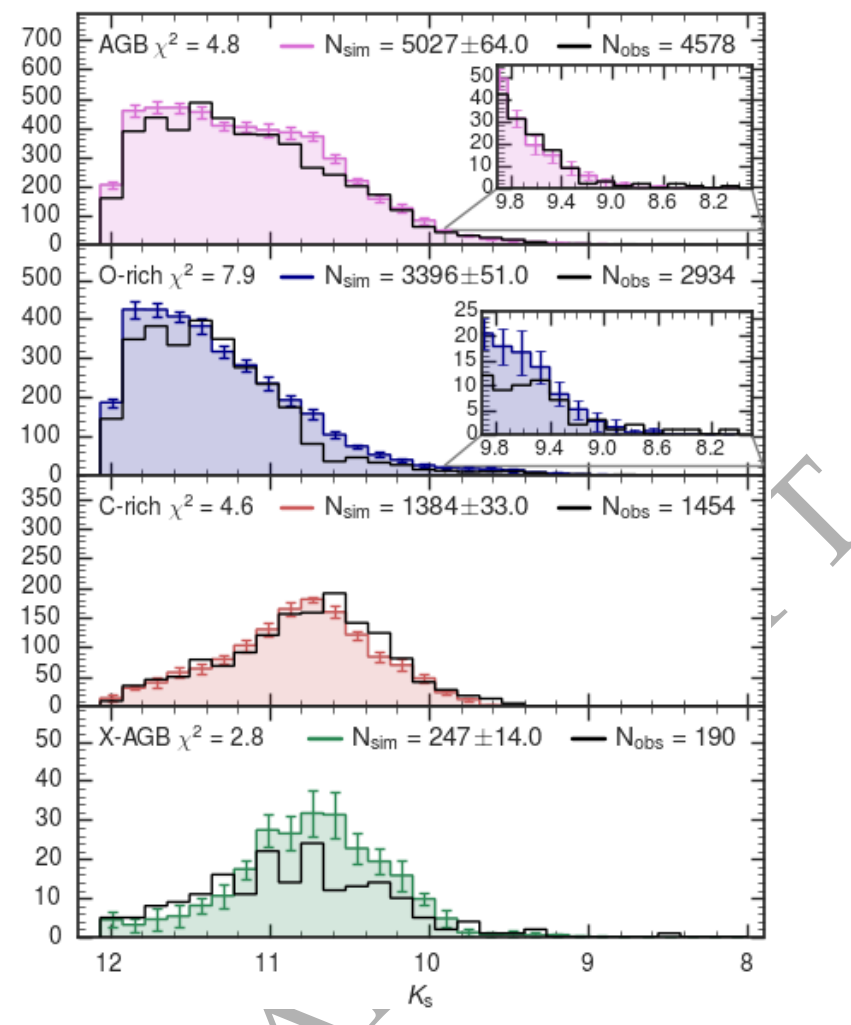

Figure 9. Same as Fig. 8, but for $\mathrm{S}_{-} 37$ in which $\lambda_{\max }^{*}$ is lowered from 0.7 to 0.5 .

according to their classification in $\mathrm{C}-, \mathrm{O}-, \mathrm{X}-$, and a-AGB stars, whereas the synthetic stars are colour-coded according to the predicted $\mathrm{C} / \mathrm{O}$ ratio. As described in Sect. 4.3, the colours of C-rich stars are in better agreement with the observations, when the effects of new opacity data are taken into account. In this case, the distribution of simulated Crich stars matches the observed $K_{\mathrm{S}}$ vs. $J-K_{\mathrm{S}}$ colours, in particular for stars brighter than $K_{\mathrm{S}} \approx 11 \mathrm{mag}$, which extend to the line that approximately separates C- and X-AGB stars. The insets highlight the CMD region in which $\mathrm{C}$ - and O-rich stars cannot be distinguished with the 2MASS photometry alone. The predicted distribution of $\mathrm{C}$ - and O-rich stars in this region is in fair agreement with the observed one. A slight shift towards redder colours is visible in the distribution of faint O-rich stars. As discussed in Sect. 2.4, this discrepancy does not affect the results of our calibration.

\subsection{MASS and Spitzer luminosity functions}

We test the performance of the best-fitting set S_37 by comparing the predicted and observed LFs in the 2MASS and Spitzer bands available in the SAGE-LMC catalogue. Fig. 13 shows such comparison for the AGB sample and for each class of AGB stars in the 2MASS $J, H, K_{\mathrm{S}}$ filters, and the Spitzer [3.6], [4.5], [5.8], [8.0] filters. In general, the synthetic LFs are in agreement with the observed ones, with some exceptions. Specifically, the predicted C- and X-AGB LFs in the Spitzer bands are shifted to fainter and brighter magnitudes respectively. As discussed by Pastorelli et al. (2019) for the SMC, where we the same kind of discrepancy is found 

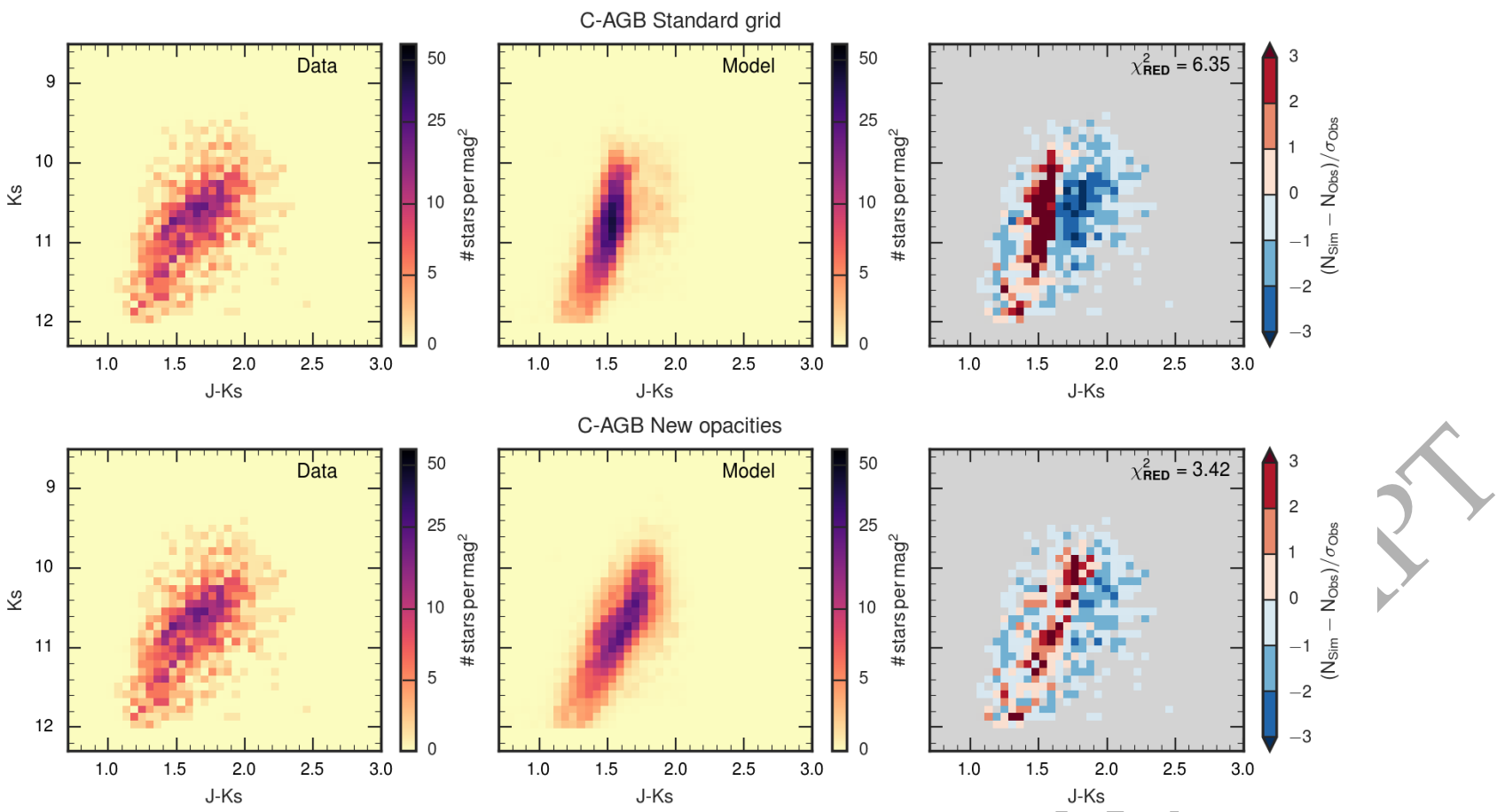

Figure 11. Comparison between the C-rich Hess diagrams calculated on the basis of the standard spectral grid by Aringer et al. (2016, top panels) and the new grid based on new molecular opacity data (bottom panels). The results from the best-fitting model S_37 (middle panels) are shown together with the observed diagram (left panels). The map of the fractional difference between observed and simulated number counts, normalized to $1 \sigma$ of the observed counts, is also plotted (right panels), along with the values of the $\chi^{2}$.
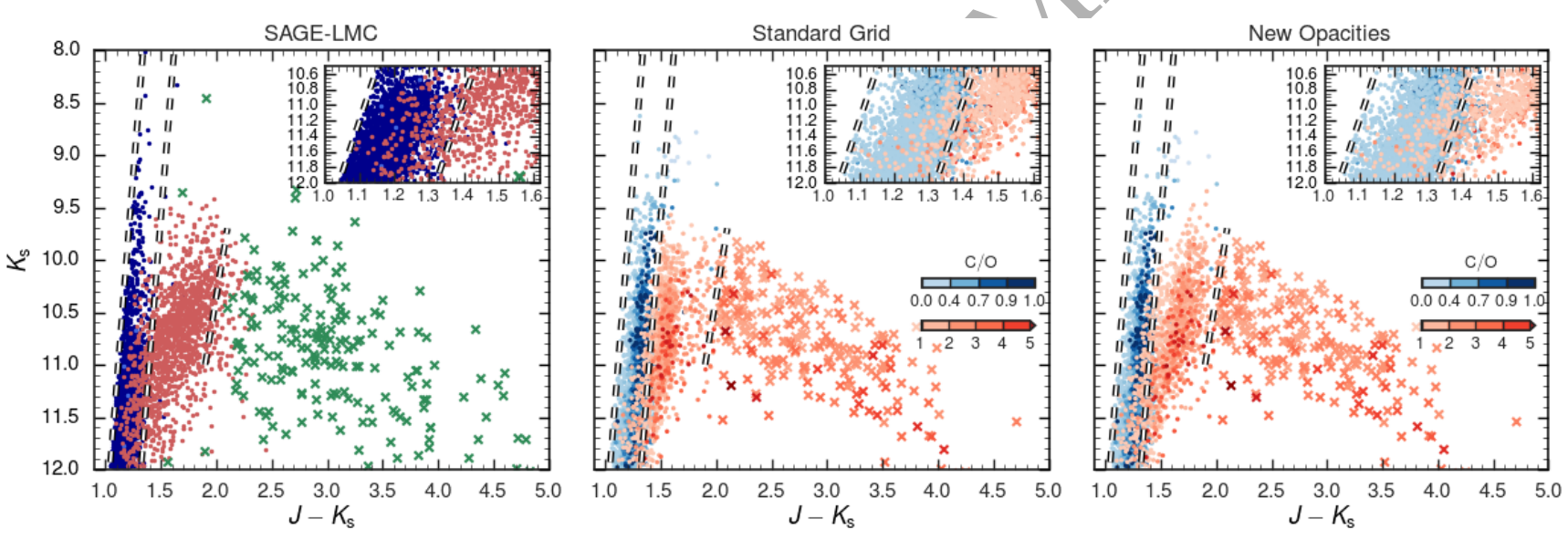

Figure 12. Left panel: observed $K_{\mathrm{S}}$ vs. $J-K_{\mathrm{S}}$ CMD with stars colour-coded according to the their classification in O-rich (blue), aAGB (light blue), C-rich (red) and X-AGB (green crosses). Middle and right panels: simulated CMDs from the best-fitting set S_37 calculated with the standard spectral grid by Aringer et al. (2016) and the grid based on new molecular opacity data. The simulated stars are colour-coded according to the predicted $\mathrm{C} / \mathrm{O}$ ratio. The insets show the CMD region where the O-rich and C-rich stars cannot be distinguished in the $K_{\mathrm{s}}$ vs. $\boldsymbol{J}_{-} K_{\mathrm{s}}$ CMD. The dashed lines are the photometric criteria used to select the observed O- and C-rich stars (Boyer et al. 2011). A third line shows the approximate separation between C- and X-AGB stars.

(see their figure 21), the differences in the X-AGB LFs do not impact our results as the percentage of X-AGB stars is less than 6 per cent of the total AGB sample. However, these discrepancies may be used to improve the circumstellar dust treatment and to test the mass-loss prescriptions in the advanced stages of TP-AGB evolution. We plan to address this point in a future study.

\section{DISCUSSION}

In the following we discuss the main relevant implications expected from the present calibration. We discuss the predictions of the set S_37 in terms of initial masses of C-rich stars, mass-loss rate distributions, TP-AGB lifetimes, and contribution of TP-AGB stars to the integrated luminosity. 

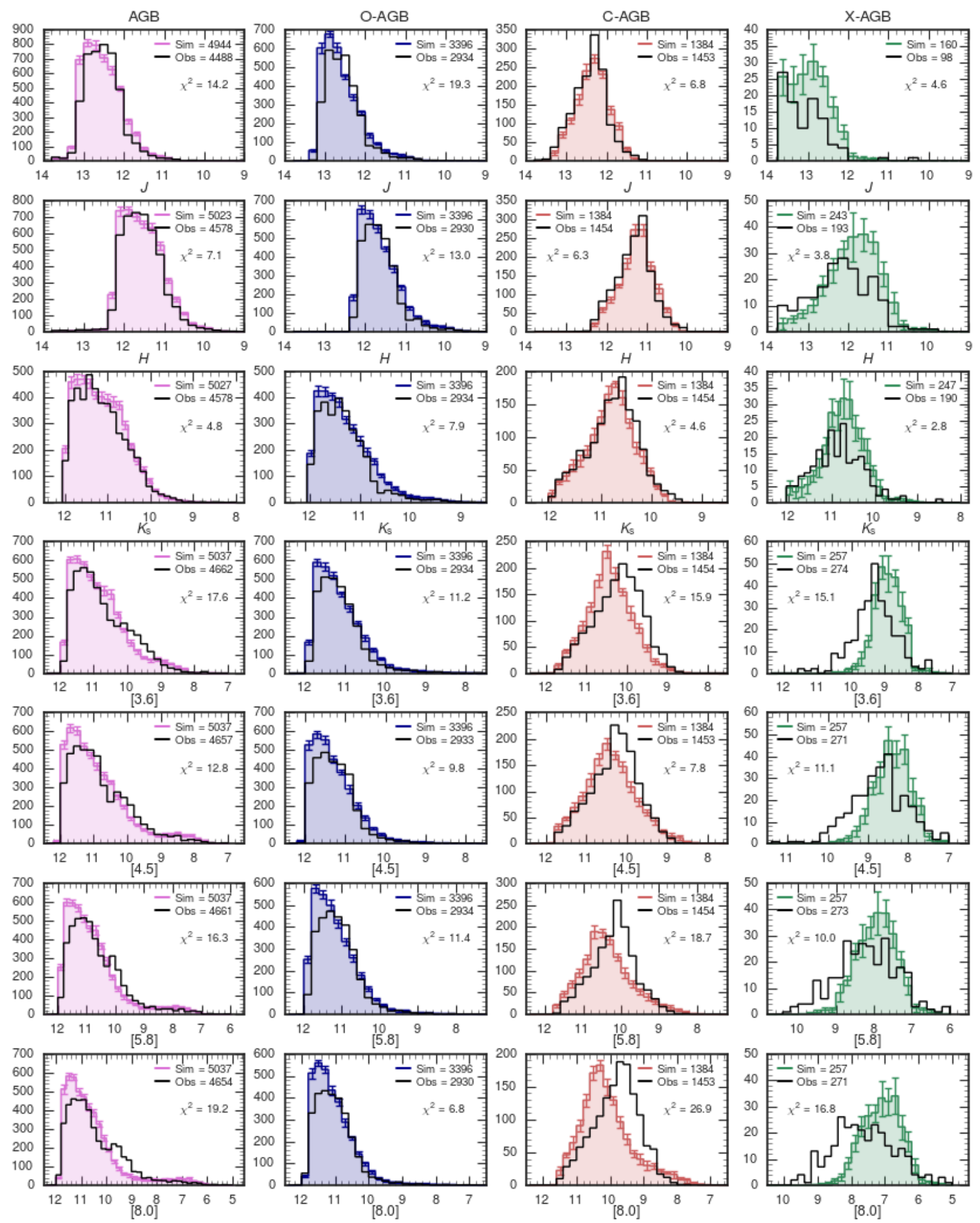

Figure 13. Comparison between the synthetic LFs obtained from the best-fitting model S_37 and the observed LFs in the 2MASS and Spitzer filters, going from shorter (top panels) to longer wavelengths (bottom panels). 


\subsection{The domain of carbon stars in the LMC}

Fig. 14 shows the predicted ranges of initial masses and bolometric magnitudes of C-rich stars for our best-fitting models, namely the set $\mathrm{S} \_35$ with $Z_{\mathrm{i}}=0.004$ based on the SMC calibration and the set $\mathrm{S}_{-} 37$ with $Z_{\mathrm{i}}=0.008$ based on the LMC calibration. The C-rich star domain extends from $M_{\mathrm{i}} \approx 1.4 \mathrm{M}_{\odot}$ to $M_{\mathrm{i}} \approx 2.8 \mathrm{M}_{\odot}$ at $Z_{\mathrm{i}}=0.004$ and from $M_{\mathrm{i}} \approx 1.7 \mathrm{M}_{\odot}$ to $M_{\mathrm{i}} \approx 3 \mathrm{M}_{\odot}$ at $Z_{\mathrm{i}}=0.008$. The minimum mass for producing carbon stars is found to decrease with decreasing metallicity, a finding that supports theoretical trends in the literature (e.g. Marigo \& Girardi 2007; Cristallo et al. 2011, 2015; Ventura et al. 2013).

It is now useful to compare the 3DU properties predicted by available full AGB models in the literature with the results of our calibration, focusing on the metallicity $Z_{\mathrm{i}}=0.008$ (or similar) which characterizes most of the carbon stars in the LMC (see Fig. 6). As shown in Fig. 15, the efficiency of the $3 \mathrm{DU}$ is still affected by significant differences from author to author, mostly evident for $M_{\mathrm{i}} \gtrsim 2 \mathrm{M}_{\odot}$. Models by Stancliffe et al. (2005) and Karakas (2010) correspond to the largest $\lambda_{\max }$ which approaches $\approx 1$ for $M_{\mathrm{i}} \gtrsim 3 \mathrm{M}_{\odot}$, while considerably lower values $\left(\lambda_{\max } \simeq 0.5-0.7\right)$ are predicted by Cristallo et al. (2011) and Ventura \& D'Antona (2009). In particular, at increasing $M_{\mathrm{i}}$, the parameter $\lambda_{\max }$ declines down to zero in Ventura \& D'Antona (2009) models, contrarily to the findings of Stancliffe et al. (2005) and Karakas (2010). Our calibrated relation for $\lambda_{\max }$ presents a trend closer to the results of Cristallo et al. (2011) and Ventura \& D'Antona (2009) but shifted to lower values.

Concerning the initial mass range of carbon stars the situation is illustrated in Table 5. Let us denote with $\mathbf{M}_{\mathrm{Cstar}}^{\mathrm{min}}$ and $\mathbf{M}_{\text {Cstar }}^{\max }$ the minimum and maximum initial mass for carbon star formation. We see that $\mathrm{M}_{\text {Cstar }}^{\min }$ at $Z_{\mathrm{i}}=0.008$ varies from $1 \mathrm{M}_{\odot}$ to $2.4 \mathrm{M}_{\odot}$. This is a notable scatter since the difference in mass translates into a wide age range, from $\approx 9.4$ Gyr to $\approx 0.7$ Gyr. The upper limit $\mathrm{M}_{\mathrm{Cstar}}^{\max }$ is found to vary between $\approx 3 \mathrm{M}_{\odot}$ to $\approx 5 \mathrm{M}_{\odot}$, which corresponds to an age interval from $\approx 0.4 \mathrm{Gyr}$ to $\approx 0.1 \mathrm{Gyr}$. We note that our results for $\mathrm{M}_{\mathrm{Cstar}}^{\max } \simeq 3 \mathrm{M}_{\odot}$ agree with the predictions of Stancliffe et al. (2005), Cristallo et al. (2011) and Dell'Agli et al. (2015).

In summary, our calibration indicates that the $3 \mathrm{DU}$ in TP-AGB LMC stars (with $Z_{\mathrm{i}}=0.008$ ) has an efficiency not exceeding $\lambda \approx 0.5$ at all initial masses. This result conflicts with the predictions of some AGB models (e.g., Stancliffe et al. 2005; Karakas 2010) in which $\lambda$ is much higher. The bulk of C-stars in the LMC should have ages between $\approx 1.7\left(\mathrm{M}_{\mathrm{Cstar}}^{\min } \simeq 1.7 \mathrm{M}_{\odot}\right)$ and $\approx 0.4 \mathrm{Gyr}$ $\left(\mathrm{M}_{\mathrm{Cstar}}^{\min } \simeq 3.0 \mathrm{M}_{\odot}\right)$.

Finally we note that C-stars with $M_{\mathrm{i}}<1.7 \mathrm{M}_{\odot}$ are expected to be present within the AGB population of the LMC, but these should have a lower metallicity $\left(Z_{\mathrm{i}}<0.008\right)$ according to the AMR. Our best-fit simulations indicate that C-stars exist down to $M_{\mathrm{i}} \approx 1.4 \mathrm{M}_{\odot}$ and $Z_{\mathrm{i}} \approx 0.004$. Similarly, the most massive carbon stars are found to have $M_{\mathrm{i}} \approx 3.2 \mathrm{M}_{\odot}$ and metallicity $Z_{\mathrm{i}} \approx 0.01$.

At this point, one may wonder if these mass ranges agree with those inferred from the presence of C-rich stars in LMC star clusters. For instance, the classical paper by Frogel et al. (1990) mentions $3-5 \mathrm{M}_{\odot}$ as the maximum mass for the formation of $\mathrm{C}$ stars in the LMC, and does not find
Table 5. Initial mass limits for C-star formation

\begin{tabular}{lccc}
\hline Reference & $\begin{array}{c}\mathrm{M}_{\mathrm{Cstar}}^{\min } \\
{\left[\mathrm{M}_{\odot}\right]}\end{array}$ & $\begin{array}{c}\mathrm{M}_{\mathrm{Cstar}}^{\max } \\
{\left[\mathrm{M}_{\odot}\right]}\end{array}$ & $Z_{\mathrm{i}}$ \\
\hline Stancliffe et al. (2005) & 1.00 & 3 & 0.008 \\
Weiss \& Ferguson (2009) & 1.00 & 5 & 0.008 \\
Karakas (2010) & 1.75 & 4 & 0.008 \\
Cristallo et al. (2011) & 1.50 & 3 & 0.008 \\
Dell'Agli et al. (2015) & 1.25 & 3 & 0.008 \\
Pignatari et al. (2016) & 1.65 & 4 & 0.01 \\
Choi et al. (2016) & 2.4 & 3.2 & $0.008^{*}$ \\
Our calibration & 1.70 & 3 & 0.008 \\
\hline
\end{tabular}

${ }^{*}$ The MIST models for a metallicity $[\mathrm{Fe} / \mathrm{H}]=-0.25$ are obtained through the web-interface at http://waps.cfa.harvard.edu/MIST/interp_tracks.html

any C-rich stars among the oldest clusters corresponding to type VII in the Searle et al. (1980) classification (ages, larger than a few Gyr). These observations suggest constraints to the minimum and maximum masses for the formation of C-rich stars; however they are based on rough and largely outdated estimates of ages and turn-off masses, $M_{\mathrm{TO}}$, for the hosting star clusters. As already mentioned in Paper I, this analysis is better done using cluster ages based only on isochrone fitting applied to high-quality photometry from the Hubble Space Telescope (HST). A quick revision of the tables in Girardi \& Marigo (2007) - which includes the additional list of cool giants in MC clusters from van Loon et al. (2005) - reveals that:

(i) NGC 1850, with $M_{\mathrm{TO}}>5.5 \mathrm{M}_{\odot}$ (and hence evolved stars in which HBB is fully operating to prevent the formation of C-rich stars), is the youngest LMC cluster to contain a C-rich star within its core radius. It is followed by the 200-Myr old LMC cluster HS 327-E $\left(M_{\mathrm{TO}} \sim 4 \mathrm{M}_{\odot}\right)$ which contains both an obscured carbon star and an $\mathrm{OH} / \mathrm{IR}$ star (van Loon et al. 2001).

(ii) The next in the list of youngest clusters to contain C stars is either NGC 1987 or NGC 2209, with $M_{\text {TO }}$ slightly below $2 \mathrm{M}_{\odot}$, and both with several C-rich stars;

(iii) the oldest one with a significant population of C-rich stars (about 10) is NGC 1978 with 2.2 Gyr and $M_{\mathrm{TO}} \approx$ $1.45 \mathrm{M}_{\odot}$ (Mucciarelli et al. 2007, and Chen et al., in preparation);

(iv) The oldest one with a modest population of $\mathrm{C}$ stars (just one) appears to be NGC 2121, for which HST data provides ages between 2.5 Gyr (Martocchia et al. 2019) and 3.2 Gyr (Rich et al. 2001; Li \& de Grijs 2019), and hence a turn-off mass just slightly smaller than NGC 1978;

(v) The age sequence is followed by the well-known gap in the history of cluster formation in the LMC - that means, a lack of clusters with ages from this limit up to 9 Gyr (see e.g. Sarajedini 1998; Rich et al. 2001; Piatti et al. 2002a,b, for a more complete discussion) - and then by a handful of very old and populous globular clusters which do not contain (nor are expected to contain) C-rich stars.

The C star in NGC 1850 deserves some discussion, because of its unusual age, and because this cluster is representative of more recent changes in the interpretative scenario of the CMDs of Magellanic Cloud star clusters. First of all, one might wonder if its C star (2MASS J05085008-6845188, cf. Boyer et al. 2013) is a chance alignment of a field star, or 

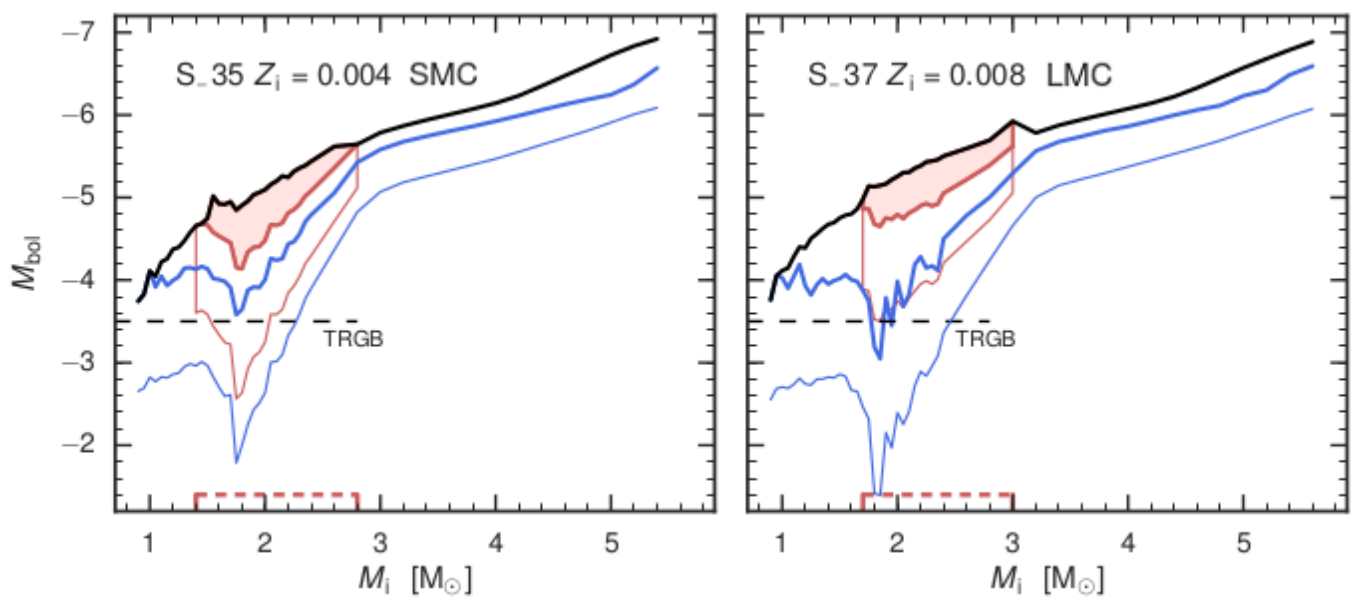

Figure 14. Bolometric magnitudes as a function of $M_{\mathrm{i}}$ for a few relevant transition stages: the first TP (blue), the transition from the O-rich to the C-rich domain (red), and the AGB tip (black). The thick solid lines connect the quiescent stages just prior to the occurrence of TPs, while thin solid lines correspond to the faintest luminosities reached during the post-flash low-luminosity dips. Results are shown for the TP-AGB sets S_35 at $Z_{\mathrm{i}}=0.004$, calibrated in the SMC, and S_37 at $Z_{\mathrm{i}}=0.008$ (LMC calibration). The range of initial masses for the formation of C-rich stars is shown by the horizontal red-dashed lines.

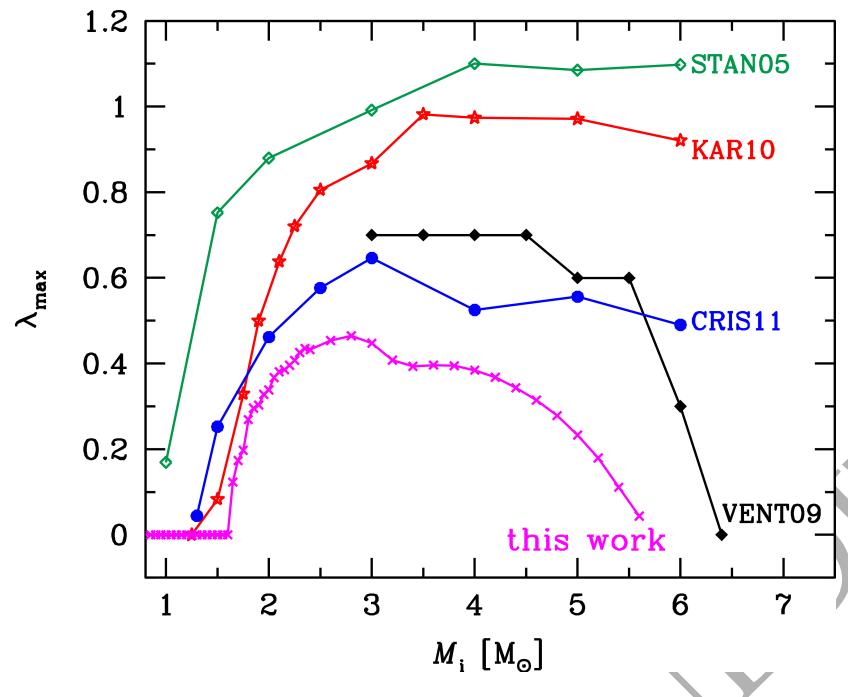

Figure 15. Maximum efficiency of the $3 D U$ as a function of the initial stellar mass, predicted by AGB models available in the literature, namely: Stancliffe et al, (2005) (green), Karakas (2010) (red), Cristallo et al. (2011) (blue), Ventura \& D'Antona (2009) (black). Our calibrated values are shown for comparison (magenta). All cases refer to $Z_{1}=0.008$, except for Ventura \& D'Antona (2009) models that correspond to $Z_{\mathrm{i}}=$ 0.006 . Note the substantial differences from author to author for $M_{\mathrm{i}}>2 \mathrm{M}_{\odot}$.

a true cluster member which already ended its HBB phase, and then underwent a late transition to the C-rich phase after losing a significant fraction of its initial stellar envelope. This latter scenario applies to the obscured carbon star in HS 327-E, which also contains the OH/IR star IRAS 052986957 van Loon et al. (2001). The main problem in this interpretation, however, is that the C star in NGC 1850 has a spectrum typical of most optically-visible $\mathrm{C}$ stars in the LMC field, with a 2MASS $K_{\mathrm{S}}=10.818$ and $J-K_{\mathrm{S}}=1.651$. Second, it is worth noticing that there are indications of a significant dispersion in the rotational velocities of stars at the turn-off of NGC 1850 (Bastian et al. 2017). This causes significant uncertainty in the initial masses inferred for its post-main sequence stars. For instance, Bastian et al. (2017) estimate $\log (\operatorname{age} / \mathrm{yr})=7.9$ and a turn-off mass of $4.86 \mathrm{M}_{\odot}$, while Johnston et al. (2019) suggest that $\log (\mathrm{age} / \mathrm{yr})$ can be as high as 8.27 and turn-off masses be as low as 1.9-4.0 $\mathrm{M}_{\odot}$.

If we disregard the single C-rich star in NGC 1850 and the obscured one in HS 327-E, we are left with firm detections of C-rich stars only in LMC clusters with turn-off masses in the narrow interval from about 1.4 to $2 \mathrm{M}_{\odot}$. The observed interval is probably very much affected by the natural scarcity of TP-AGB stars in the youngest clusters with higher turn-off masses, and might be affected by the scarcity of star clusters in the age range corresponding to the lowest turn-off mass. Star clusters in the LMC, such as NGC 1978 and NGC 2121, indicate that the minimum mass to form C-rich stars should be around $M_{\mathrm{i}} \approx 1.45 \mathrm{M}_{\odot}$, which is lower than the predicted limit of $M_{\mathrm{i}} \approx 1.7 \mathrm{M}_{\odot}$ at $Z_{\mathrm{i}}=0.008$. However, the discrepancy is only apparent since the metallicity of NGC 1978 is $Z_{\mathrm{i}} \approx 0.005$, as suggested by the analysis of nearIR CMDs of the cluster (Chen et al., in prep.). Therefore the observational indication is consistent with our expectations at $Z_{\mathrm{i}}=0.004$.

\subsection{Comparing the C-rich LFs in the LMC and SMC}

At this point it is interesting to compare the C-rich LFs in the Magellanic Clouds. The observed distributions in the absolute $K_{\mathrm{S}}$ magnitude are presented in the top panel of Fig. 16. Distances and reddening corrections are taken from the results of the SFH recovery, described in Sect. 2.1 for the LMC and in Pastorelli et al. (2019) for the SMC. We should recall here that the observed sample of carbon stars in the LMC is extracted from a selection of VMC tiles which do not cover the entire galaxy (see Sect. 2.1). Anyhow, we 

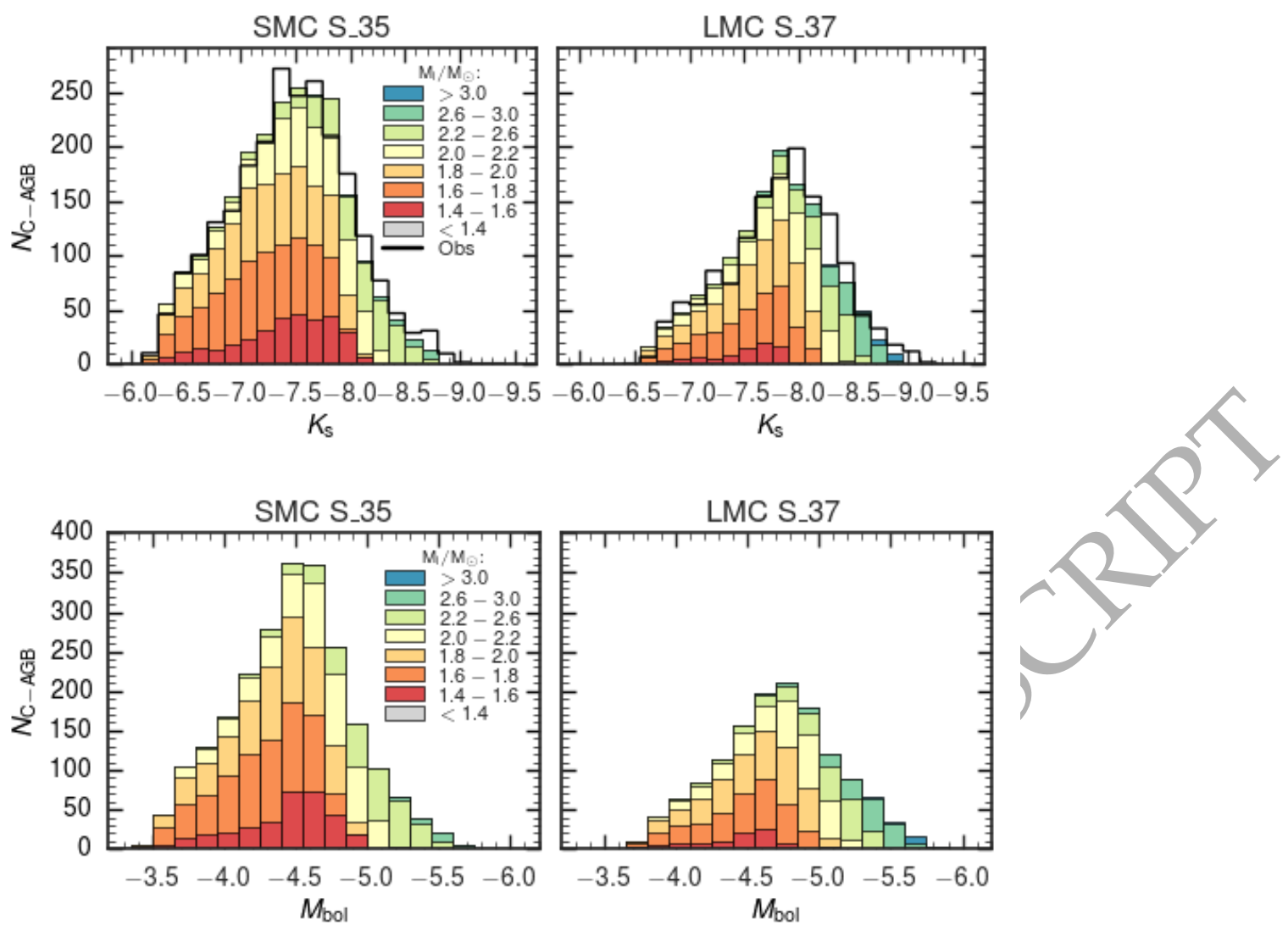

Figure 16. Top panel: observed C-rich star luminosity functions in the absolute $K_{\mathrm{S}}$ magnitude (black solid histograms). Best-fitting models (set S_35 for the SMC and set S_37 for the LMC) are over-plotted as stacked histograms binned as a function of the initial stellar mass. Bottom panel: theoretical C-rich star luminosity distributions in absolute bolometric magnitude.

can derive some useful indications coupling observations and best-fitting models.

In the absolute $K_{\mathrm{S}}$ magnitude the C-rich LFs present a similar morphology, but with two main evident differences. First, in the LMC the peak is shifted towards brighter magnitudes (by $\approx 0.6 \mathrm{mag}$ ) compared with the SMC. Similarly, a small magnitude shift in the $J$-band C-rich $L F$ peaks of the two galaxies has been recently reported and analysed by Ripoche et al. (2020, see also Fig. 4.5 for the LFs in 2MASS filters). We interpret such a difference as due to the characteristics of the 3DU which is, on average, somewhat less efficient in the LMC, likely driven by a metallicity effect. At the same time the minimum mass for carbon star formation is expected to be larger at higher metallicity (see Sect. 5.1). Second, the observed C-rich LF extends to fainter $K_{\mathrm{S}}$ magnitudes in the SMC, since at lower metallicity the transition to the carbon star domain takes place earlier on the TP-AGB, that is at fainter magnitudes (see Fig. 14).

Similar considerations apply to the predicted C-rich LFs in bolometric magnitude (bottom panel of Fig. 16): in the SMC the peak appears located at $M_{\text {bol }} \approx-4.45$, while the maximum of the LMC distribution is at $M_{\mathrm{bol}} \approx-4.75$.

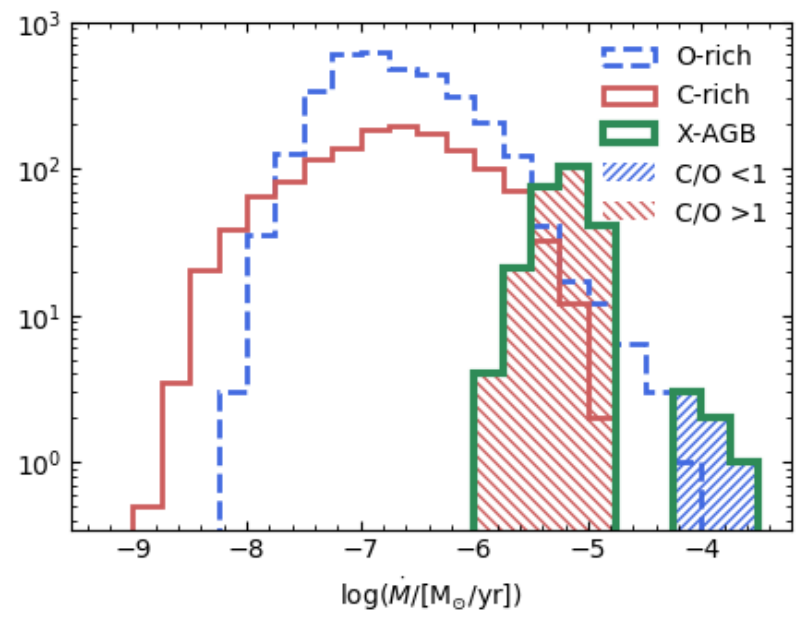

Figure 17. Predicted mass-loss rates distributions for the O-, C-, and X-AGB synthetic populations from the set S_37. The X-AGB stars with $\mathrm{C} / \mathrm{O}>1$ and those with $\mathrm{C} / \mathrm{O}<1$ are shown as red and blue hatched regions, respectively, as indicated in the legend.

\subsection{Mass-loss rates}

The distributions of the mass-loss rates of the O-, C-, and $\mathrm{X}$-AGB synthetic populations from the set $\mathrm{S} \_37$ are shown in 
Fig. 17. These results are similar to our previous SMC analysis, and are in broad agreement with the results of the SED fitting works by (Nanni et al. 2019; Groenewegen \& Sloan 2018). The majority of C- and O-rich stars have mass-loss rates in the range $\approx 10^{-8}-10^{-6} \mathrm{M}_{\odot} \mathrm{yr}^{-1}$. The X-AGB stars show the highest values of mass-loss rates, ranging from $\approx 10^{-6}-10^{-4} \mathrm{M}_{\odot} \mathrm{yr}^{-1}$. Specifically, the highest mass-loss rates are achieved by obscured O-rich AGBs experiencing $\mathrm{HBB}$ and with initial masses larger than $3 \mathbf{M}_{\odot}$. The percentage of $\mathrm{O}$ - and $\mathrm{C}$-rich $\mathrm{X}-\mathrm{AGB}$ stars in our simulations are $\approx 2$ and $\approx 98$ per cent respectively. This is in agreement with the general idea that the X-AGB sample is mainly populated by C-rich stars (van Loon et al. 2005; Dell'Agli et al. 2015)

\subsection{Predicted TP-AGB lifetimes}

The reproduction of the observed star counts allows us to directly constrain the duration of the TP-AGB phase in the metallicity range considered. The lifetimes of TP-AGB stars are closely linked to their contribution to the integrated light of galaxies and to the total ejecta that they can return to the interstellar medium.

In Fig. 18, we show the lifetimes for O- and C-rich stars as a function of initial metallicity, which result from our SMC and LMC calibration. Specifically, we plot the predicted lifetimes extracted from the COLIBRI set S_35 for $Z_{\mathrm{i}}=0.0005,0.001,0.004$ (SMC calibration) and from the $\mathrm{S} \_37$ for $Z_{\mathrm{i}}=0.008,0.014,0.02$ (LMC calibration). We caution that the results for solar-like metallicities, $Z_{\mathrm{i}} \geq 0.014$ need to be further checked, and likely revised, with the help of observations that better probe this metallicity regime (e.g. AGB stars in M31, or the IFMR in Galactic open clusters). This work is underway.

The O-rich lifetimes tend to increase with the initial metallicity. This is the combined result of the pre-dust mass-loss prescription, which is efficient in low-mass lowmetallicity AGB stars, and the longer duration of the O-rich stages given that it is more difficult to form carbon stars at higher $Z_{\mathrm{i}}$. As to the C-rich lifetimes, a mirror-like trend is predicted. At larger $Z_{\mathrm{i}}$, on average, the duration is shortened since the TP-AGB phase is dominated by the O-rich stages. Note that the peak in the lifetimes of C-rich stars tends to shift to larger initial masses at increasing $Z_{\mathrm{i}}$

\subsection{TP-AGB contribution to integrated luminosity}

As one of the main motivations for providing calibrated TPAGB evolutionary tracks and isochrones resides on the impact of this phase to the integrated light of galaxies, we compute the predicted contribution of TP-AGB stars to the luminosity of simple stellar populations (SSPs). Fig. 19 shows this contribution, in both bolometric and $K$-band light, at $Z_{\mathrm{i}}=0.004$ and $Z_{\mathrm{i}}=0.008$. The evolutionary tracks at $Z_{\mathrm{i}}=0.004$ and $Z_{\mathrm{i}}=0.008$ used to compute the TP-AGB contributions to the SSPs are calibrated in the SMC (Paper I) and in the LMC (this work), respectively.

We note that the spike at $\log ($ age $/$ yr $) \simeq 9.2$ corresponds to the occurrence of the 'AGB-boosting' effect, which is intimately linked to the physics of the stellar structure (refer to Girardi et al. 2013, for a thorough discussion).
In general, our predicted TP-AGB contribution peaks around $\sim 1$ Gyr and does not exceed $\simeq 50-55$ per cent in the $K$-band luminosity ( $\simeq 20-25$ per cent in bolometric luminosity $)$. For ages younger than $\log ($ age $/ \mathrm{yr}) \approx 8.5$ and older than $\log ($ age $/ y r) \approx 10$, the predicted TP-AGB contribution is almost entirely due to O-rich stars and increases with metallicity, as the O-rich lifetimes. The predicted contribution around the peak slightly increases from $Z_{\mathrm{i}}=0.004$ to $Z_{\mathrm{i}}=0.008$ (for both $K$-band and bolometric luminosity).

We also compare our results to the popular stellar population synthesis models by Bruzual \& Charlot (2003, BC03) and Maraston (2005, M05). Our models predict a lower contribution with respect to M05 for which the peak in bolometric luminosity reaches 30 per cent, and it is as high as 80 per cent in the $K$-band at $Z_{\mathrm{i}}=0.004$. Furthermore, the peak in M05 is shifted to younger ages with respect to both our results and $\mathrm{BC} 03$. In this respect, we note that such differences are expected to be mitigated if we consider the work by Noël et al. (2013) which suggest a significant reduction of the TP-AGB contribution in M05 models based on a new analysis of the integrated colours of MCs' clusters. The results of our models are in general closer to BC03. The main differences in this case are the higher contribution predicted by BC03 for $\log ($ age $/ \mathrm{yr}) \lesssim 8.5-8.7$ and $\log ($ age $/ \mathrm{yr}) \gtrsim 9.5$ for both the bolometric and $K$-band luminosity.

\section{CONCLUSIONS}

In this work we extend the calibration of COLIBRI TPAGB models to the LMC. To this purpose, we couple high-quality data of the AGB population in the LMC, with detailed stellar population synthesis simulations computed with the TRILEGAL code. We make use of the catalogue of AGB stars photometrically classified by Boyer et al. (2011), and complemented with spectroscopic information from Boyer et al. (2015b); Groenewegen \& Sloan (2018); Kontizas et al. (2001); Skiff (2014). We revise the classification of a-AGB stars by using their location in the Gaia2MASS diagram, that allows a better separation between O- and C-rich stars with respect to the 2MASS and Spitzer colours alone. The TRILEGAL simulations are based on the spatially resolved SFH derived from the deep near-infrared data of the VMC survey. Despite the partial coverage of the current SFH data, and the constraints on the quality of the $\mathrm{SFH}$ solutions (i.e. we consider only VMC subregions for which the RGB number counts are reproduced within $3 \sigma$ ) the observed sample contains a statistically significant number of AGB stars $(\approx 4600)$. We compare the observed and simulated $K_{\mathrm{S}}$-band LFs of the O-, C-, X-AGB samples to investigate the $3 \mathrm{DU}$ at LMC-like metallicities.

We reproduce the LMC photometry with the bestfitting set (S_37), that has the same mass-loss prescription as the starting set (S_35), but a reduced efficiency of the $3 \mathrm{DU}$ for metallicities higher than $Z_{\mathrm{i}}=0.008$. Such a decreasing trend of the 3DU efficiency is in line with the results of the full TP-AGB models by Cristallo et al. (2011) and Ventura \& D'Antona (2009).

As new molecular linelists for species relevant to the C-rich star opacity recently became available, a new set of COMARCS models is currently being calculated. In this work we test how the new opacity data affect the predicted colours 


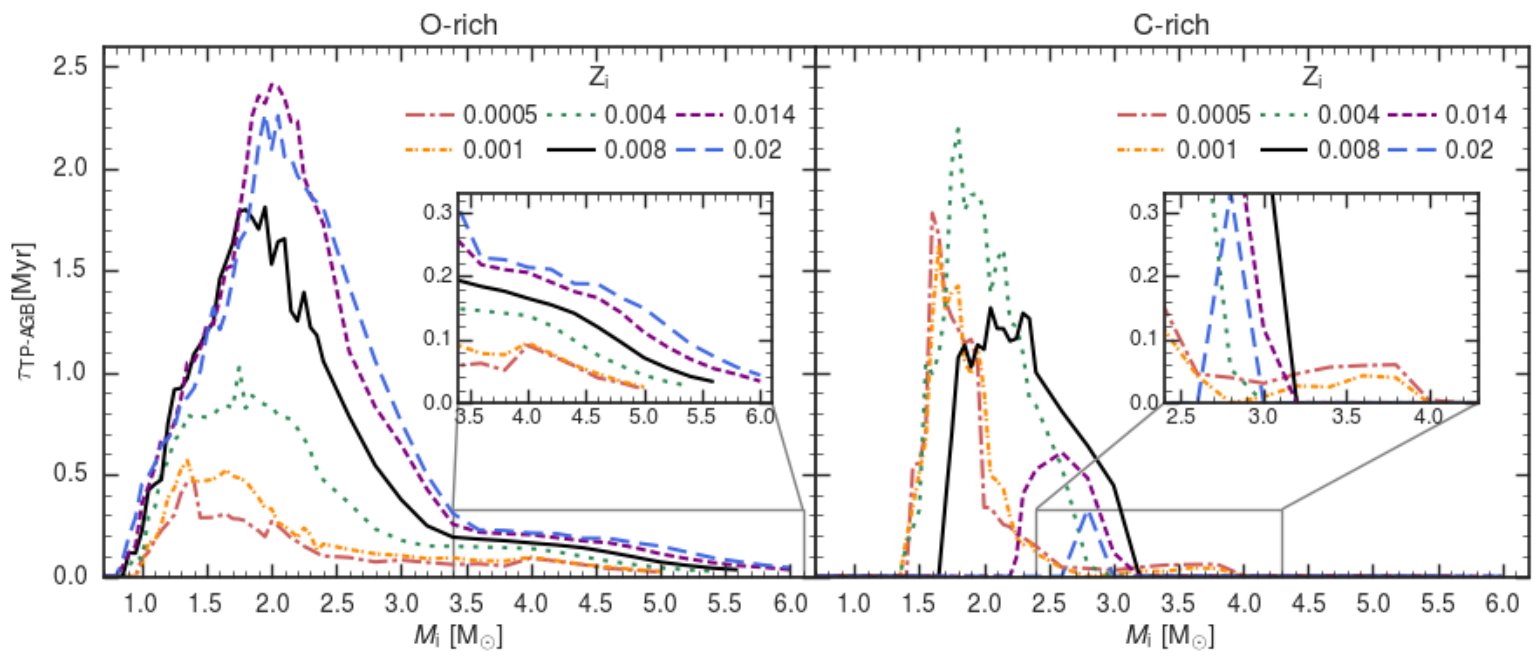

Figure 18. TP-AGB lifetimes of $\mathrm{O}$ - and C-rich stars for selected values of initial metallicities as predicted by $\mathrm{S}_{-} 35$ for $Z_{\mathrm{i}}=$ $0.0005,0.001,0.004$ (SMC calibration Pastorelli et al. 2019) and by the $S_{-} 37$ for $Z_{i}=0.008,0.014,0.02$ (LMC calibration, this work). The lifetimes for the more massive stars are shown in the two insets of each panel. As explained in the text, the lifetimes for $Z_{\mathrm{i}} \geq 0.014$ are likely to be revised.

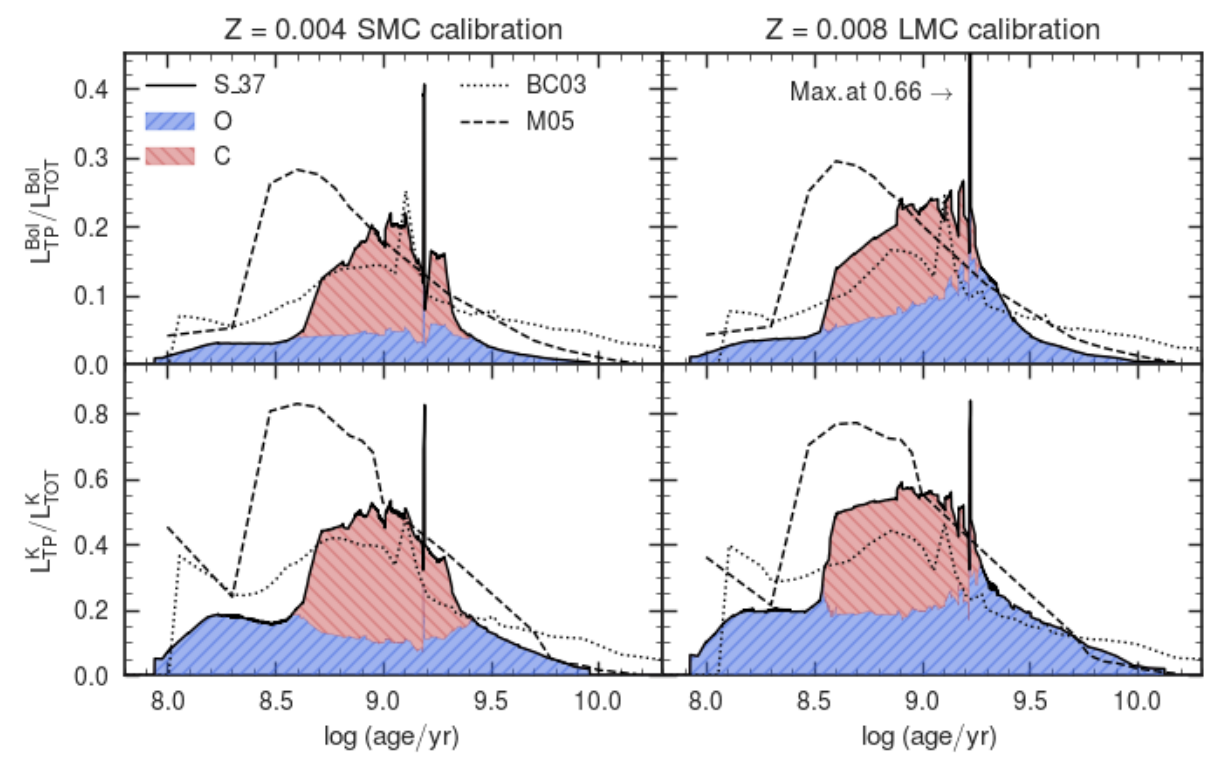

Figure 19. Predicted contribution of TP-AGB stars to the total bolometric luminosity (upper panel) and the $K$-band luminosity (lower panel), as a function of age, from the COLIBRI set $\mathrm{S}_{-3} 3$ at $Z_{\mathrm{i}}=0.004$ and $Z_{\mathrm{i}}=0.008$ (solid black lines). The results for $Z_{\mathrm{i}}=0.004$ are based on the SMC calibration from Pastorelli et al. 2019, while the results for $Z_{i}=0.008$ are from the LMC calibration discussed in this work. The red and blue hatched regions correspond to the contributions of C- and O-rich stars, respectively. We compare our results with Maraston (2005, M05) and Bruzual \& Charlot (2003, BC03) models, which are shown as black dashed lines and black dotted lines respectively.

of C-rich stars. We find that a significant improvement in the LMC $J-K_{\mathrm{S}}$ colour distribution and $K_{\mathrm{S}}$-LFs can be achieved with the new models. However, the new grid of COMARCS models is not yet complete and cannot be consistently used in TRILEGAL for the calculation of synthetic photometry. We also show that the new data will not affect our previous SMC calibration.

We also find a fairly good agreement between the predicted and observed LFs in the 2MASS and Spitzer filters.
We then discuss our best-fitting model in terms of carbon star formation, mass-loss rate distributions, stellar lifetimes and predicted contribution to the integrated light of SSPs. The C-rich star domain at $Z_{\mathrm{i}}=0.008$ extends from $M_{\mathrm{i}} \approx 1.7 \mathrm{M}_{\odot}$ to $M_{\mathrm{i}} \approx 3 \mathrm{M}_{\odot}$, whereas at $Z_{\mathrm{i}}=0.004$ from $M_{\mathrm{i}} \approx 1.4 \mathrm{M}_{\odot}$ to $M_{\mathrm{i}} \approx 2.8 \mathrm{M}_{\odot}$. The minimum initial mass for C-rich star formation is predicted to decrease with decreasing metallicity, in agreement with other works in the literature. Our results are in agreement with the 
mass ranges inferred from LMC clusters. The mass-loss rate distributions predicted by our best-fitting model are in broad agreement with the results of the SED studies by Groenewegen \& Sloan (2018) and Nanni et al. (2019). With respect to the SMC calibration (S_35), the best-fitting model S_37 predicts longer lifetimes for O-rich stars and shorter lifetimes for C-rich stars at $Z_{\mathrm{i}}=0.008$. The peak of the TPAGB contribution to the bolometric and $K$-band luminosity predicted by our best-fitting model $\mathrm{S} \_37$ at $Z_{\mathrm{i}}=0.008$ and ages around 1 Gyr lies in between the predictions by BC03 and M05.

New sets of PARSEC+COLIBRI stellar isochrones derived from the best-fitting model presented in this work (S_37) are available via our web interface http://stev.oapd.inaf.it/cmd. We refer to Marigo et al. (2017) for all the details concerning the construction and the format of the provided isochrones. The current stellar isochrones also include the predicted pulsation periods of long-period variables from Trabucchi et al. (2019). The new set of bolometric corrections by Chen et al. (2019) are also available, and will become the default option for subsequent works. The present calibration of COLIBRI TP-AGB models covers the sub-solar metallicity range relevant for the Magellanic Clouds $\left(Z_{\mathrm{i}}=0.0005-0.01\right)$. We provide isochrones also for higher metallicities, i.e. $Z_{i}=0.014,0.017,0.02$, which cover the solar-like metallicity range. In this respect we caution the users that these models may need to be revised with the aid of observational constraints that suitably probe the solar-metallicity regime. Work is in progress using AGB stars in M31 and the IFMR in the Milky Way. The same applies to very low metallicities using data for Local Group dwarf galaxies. Further studies are also planned for the LMC, once the new grid of COMARCS model spectra for AGB stars and the SFH of more VMC tiles become available. In particular, we will explore in more details the dependence of our calibration on the age (hence initial mass) of AGB stars by studying the LFs of regions in the Magellanic Clouds characterized by young and old stellar populations separately.

\section{ACKNOWLEDGEMENTS}

Many of us (PM, GP, LG, SB, YC, BA, MT, MATG, JD, SR) acknowledge the support from the ERC Consolidator Grant funding scheme (project STARKEY, grant agreement n. 615604). We thank C. Maraston, S. Charlot, and G. Bruzual for providing us with their stellar population synthesis models. M-RC acknowledges support the European Research Council (ERC) under the European UnionâĂŹs Horizon 2020 résearch and innovation programme (grant agreement no. 682115). AN acknowledges the support by the Centre National dâÁŹEtudes Spatiales (CNES) through post-doctoral fellowship. This work is based on the observations collected at the European Organisation for Astronomical Research in the Southern Hemisphere under ESO programme 179.B-2003. We thank the CASU and the WFAU for providing calibrated data products under the support of the Science and Technology Facility Council (STFC) in the UK. This publication makes use of data products from the Two Micron All Sky Survey, which is a joint project of the University of
Massachusetts and the Infrared Processing and Analysis Center/California Institute of Technology, funded by the National Aeronautics and Space Administration and the National Science Foundation. This work has made use of data from the European Space Agency (ESA) mission Gaia (https://www.cosmos.esa.int/gaia), processed by the Gaia Data Processing and Analysis Consortium (DPAC, https://www cosmos.esa.int/web/gaia/dpac/consortium). Funding for the DPAC has been provided by national institutions, in particular the institutions participating in the Gaia Multilateral Agreement. This research made use of Astropy, a community-developed core Python package for Astronomy (Astropy Collaboration et al. 2013, 2018) and matplotlib, a Python library for publication quality graphics (Hunter 2007).

\section{DATA AVAILABILITY}

The stellar models and isochrones generated in this research will be shared via the CMD web interface at http://stev.oapd.inaf.it/cmd. The VMC data used in this research will be soon shared by ESO via its regular data releases (see http://www.eso.org/rm/publicAccess\#/dataReleases).

The catalogues of observed AGB stars underlying this article are available from Boyer et al. (2011) at 10.26093/cds/vizier.51420103, Groenewegen \& Sloan (2018) at 10.26093/cds/vizier.36090114, Kontizas et al. (2001) at 10.26093/cds/vizier.33690932, Skiff (2014) at http://vizier.u-strasbg.fr/viz-bin/VizieR?-source=B/mk. The spectroscopic classification data presented in Boyer et al. (2015b) and used in this research were provided by M. L. Boyer by permission. Data will be shared on reasonable request to the corresponding author with permission of M. L. Boyer.

\section{REFERENCES}

Aringer B., Girardi L., Nowotny W., Marigo P., Lederer M. T., 2009, A\&A, 503, 913

Aringer B., Girardi L., Nowotny W., Marigo P., Bressan A., 2016, MNRAS, 457, 3611

Astropy Collaboration et al., 2013, A\&A, 558, A33

Astropy Collaboration et al., 2018, AJ, 156, 123

Barber R. J., Strange J. K., Hill C., Polyansky O. L., Mellau G. C., Yurchenko S. N., Tennyson J., 2014, MNRAS, 437, 1828

Bastian N., et al., 2017, MNRAS, 465, 4795

Bladh S., Eriksson K., Marigo P., Liljegren S., Aringer B., 2019, A\&A, 623, A119

Bloecker T., 1995, A\&A, 297, 727

Blum R. D., et al., 2006, AJ, 132, 2034

Boyer M. L., et al., 2011, AJ, 142, 103

Boyer M. L., et al., 2012, ApJ, 748, 40

Boyer M. L., et al., 2013, ApJ, 774, 83

Boyer M. L., et al., 2015a, ApJS, 216, 10

Boyer M. L., McDonald I., Srinivasan S., Zijlstra A., van Loon J. T., Olsen K. A. G., Sonneborn G., 2015b, ApJ, 810, 116

Boyer M. L., et al., 2017, ApJ, 851, 152

Boyer M. L., et al., 2019, ApJ, 879, 109

Bressan A., Granato G. L., Silva L., 1998, A\&A, 332, 135

Bressan A., Marigo P., Girardi L., Salasnich B., Dal Cero C., Rubele S., Nanni A., 2012, MNRAS, 427, 127 
Brooke J. S. A., Ram R. S., Western C. M., Li G., Schwenke D. W., Bernath P. F., 2014, ApJS, 210, 23

Bruzual G., Charlot S., 2003, MNRAS, 344, 1000

Caffau E., Ludwig H.-G., Steffen M., Freytag B., Bonifacio P., 2011, Sol. Phys., 268, 255

Castelli F., Kurucz R. L., 2003, in Piskunov N., Weiss W. W., Gray D. F., eds, IAU Symposium Vol. 210, Modelling of Stellar Atmospheres. p. A20

Chen Y., et al., 2019, A\&A, 632, A105

Choi J., Dotter A., Conroy C., Cantiello M., Paxton B., Johnson B. D., 2016, ApJ, 823, 102

Chubb K. L., Tennyson J., Yurchenko S. N., 2020, MNRAS, p. 221

Cioni M. R. L., et al., 2011, A\&A, 527, A116

Conroy C., 2013, ARA\&A, 51, 393

Cranmer S. R., Saar S. H., 2011, ApJ, 741, 54

Cristallo S., et al., 2011, ApJS, 197, 17

Cristallo S., Straniero O., Piersanti L., Gobrecht D., 2015, ApJS, 219,40

Cummings J. D., Kalirai J. S., Tremblay P. E., Ramirez-Ruiz E., Choi J., 2018, ApJ, 866, 21

Dalcanton J. J., et al., 2009, ApJS, 183, 67

Dalcanton J. J., et al., 2012, ApJS, 198, 6

Dell'Agli F., Ventura P., Schneider R., Di Criscienzo M., GarcíaHernández D. A., Rossi C., Brocato E., 2015, MNRAS, 447, 2992

El-Badry K., Rix H.-W., Weisz D. R., 2018, ApJ, 860, L17

Elitzur M., Ivezić Ž., 2001, MNRAS, 327, 403

Eriksson K., Nowotny W., Höfner S., Aringer B., Wachter A., 2014, A\&A, 566, A95

Ferrarotti A. S., Gail H.-P., 2006, A\&A, 447, 553

Frogel J. A., Mould J., Blanco V. M., 1990, ApJ, 352, 96

Frost C. A., Lattanzio J. C., 1996, ApJ, 473, 383

Gaia Collaboration et al., 2018, A\&A, 616, A1

Girardi L., Marigo P., 2007, A\&A, 462, 237

Girardi L., Groenewegen M. A. T., Hatziminaoglou E., da Costa L., 2005, A\&A, 436, 895

Girardi L., et al., 2010, ApJ, 724, 1030

Girardi L., Marigo P., Bressan A., Rosenfield P., 2013, ApJ, 777,142

Groenewegen M. A. T., 2006, A\&A, 448, 181

Groenewegen M. A. T., Sloan G. C., 2018, A\&A, 609, A114

Groenewegen M. A. T., de Jong T., 1993, A\&A, 267, 410

Hamedani Golshan R., Javadi A., van Loon J. T., Khosroshahi H., Saremi E., 2017, MNRAS, 466, 1764

Hashemi S. A., Javadi A., van Loon J. T., 2019, MNRAS, 483,4751

Herwig F., 2005, ARA\&A, 43, 435

Höfner S., Olofsson H., 2018, A\&ARv, 26,

Huang C. D., et al., 2018, ApJ, 857, 67

Huang C. D., et al., 2020, ApJ, 889, 5

Hunter J. D., 2007, Computing In Science \& Engineering, 9, 90

Javadi A., van Loon J. T., Khosroshahi H., Mirtorabi M. T., 2013, MNRAS, 432, 2824

Javadi A., van Loon J. T., Khosroshahi H. G., Tabatabaei F., Hamedani Golshan R., Rashidi M., 2017, MNRAS, 464, 2103

Johnston C., Aerts C., Pedersen M. G., Bastian N., 2019, A\&A, $632, \mathrm{~A} 74$

Karakas A. I., 2010, MNRAS, 403, 1413

Karakas A. I., Lattanzio J. C., 2014, Publ. Astron. Soc. Australia, 31, e030

Karakas A. I., Lattanzio J. C., Pols O. R., 2002, Publ, Astron. Soc. Australia, 19, 515

Kobayashi C., Karakas A. I., Umeda H., 2011, MNRAS, 414, 3231

Kontizas E., Dapergolas A., Morgan D. H., Kontizas M., 2001, A\&A, 369, 932

Kroupa P., 2001, MNRAS, 322, 231

Lebzelter T., Mowlavi N., Marigo P., Pastorelli G., Trabucchi M., Wood P. R., Lecoeur-Taïbi I., 2018, A\&A, 616, L13
Lewis A. R., et al., 2015, ApJ, 805, 183

Li C., de Grijs R., 2019, ApJ, 876, 94

Madore B. F., Freedman W. L., 2020, arXiv e-prints, p. arXiv:2005.10792

Maraston C., 2005, MNRAS, 362, 799

Maraston C., Daddi E., Renzini A., Cimatti A., Dickinson M., Papovich C., Pasquali A., Pirzkal N., 2006, ApJ, 652, 85

Marigo P., 2015, in Kerschbaum F., Wing R. F., Hron J., eds, Astronomical Society of the Pacific Conference Series Vol. 497, Why Galaxies Care about AGB Stars III: A Closer Look in Space and Time. p. 229 (arXiv:1411.3126)

Marigo P., Aringer B., 2009, A\&A, 508, 1539

Marigo P., Girardi L., 2007, A\&A, 469, 239

Marigo P., Girardi L., Bressan A., 1999, A\&A, 344, 123

Marigo P., Girardi L., Bressan A., Groenewegen M. A. T., Silva L., Granato G. L., 2008, A\&A, 482, 883

Marigo P., Bressan A., Nanni A., Girardi L., Pumo M. L., 2013, MNRAS, 434, 488

Marigo P., et al., 2017, ApJ, 835, 77

Marrese P. M., Marinoni S., Fabrizio M., Altavilla G., 2019, A\&A, 621, A144

Martocchia S., et al., 2019, MNRAS, 487, 5324

Masseron T., et al., 2014, A\&A, 571, A47

Mattsson L., Wahlin R., Höfner S., 2010, A\&A, 509, A14

Meixner M., et al., 2006, AJ, 132, 2268

Melbourne J., Boyer M. L., 2013, ApJ, 764, 30

Mucciarelli A., Ferraro F. R., Origlia L., Fusi Pecci F., 2007, AJ, 133, 2053

Nanni A., Marigo P., Girardi L., Rubele S., Bressan A., Groenewegen M. A. T., Pastorelli G., Aringer B., 2018, MNRAS, 473,5492

Nanni A., Groenewegen M. A. T., Aringer B., Rubele S., Bressan A., van Loon J. T., Goldman S. R., Boyer M. L., 2019, MNRAS, 487, 502

Noël N. E. D., Greggio L., Renzini A., Carollo C. M., Maraston C., 2013, ApJ, 772, 58

Olsen K. A. G., Zaritsky D., Blum R. D., Boyer M. L., Gordon K. D., 2011, ApJ, 737, 29

Pastorelli G., et al., 2019, MNRAS, 485, 5666

Piatti A. E., Sarajedini A., Geisler D., Bica E., Clariá J. J., 2002a, MNRAS, 329, 556

Piatti A. E., Sarajedini A., Geisler D., Bica E., Clariá J. J., 2002b, MNRAS, 329, 556

Pierce M. J., Jurcevic J. S., Crabtree D., 2000, MNRAS, 313, 271

Pignatari M., et al., 2016, ApJS, 225, 24

Rezaeikh S., Javadi A., Khosroshahi H., van Loon J. T., 2014, MNRAS, 445, 2214

Rich R. M., Shara M. M., Zurek D., 2001, AJ, 122, 842

Ripoche P., Heyl J., Parada J., Richer H., 2020, MNRAS, 495, 2858

Rosenfield P., et al., 2014, ApJ, 790, 22

Rubele S., et al., 2018, MNRAS, 478, 5017

Sarajedini A., 1998, AJ, 116, 738

Schneider R., Valiante R., Ventura P., dell'Agli F., Di Criscienzo M., Hirashita H., Kemper F., 2014, MNRAS, 442, 1440

Searle L., Wilkinson A., Bagnuolo W. G., 1980, ApJ, 239, 803

Skiff B. A., 2014, VizieR Online Data Catalog, p. B/mk

Skrutskie M. F., et al., 2006, AJ, 131, 1163

Sneden C., Lucatello S., Ram R. S., Brooke J. S. A., Bernath P., 2014, ApJS, 214, 26

Srinivasan S., Boyer M. L., Kemper F., Meixner M., Sargent B. A., Riebel D., 2016, MNRAS, 457, 2814

Stancliffe R. J., Izzard R. G., Tout C. A., 2005, MNRAS, 356, L1

Tennyson J., et al., 2016, J. Mol. Spectrosc., 327, 73

Trabucchi M., Wood P. R., Montalbán J., Marigo P., Pastorelli G., Girardi L., 2019, MNRAS, 482, 929

Valiante R., Schneider R., Bianchi S., Andersen A. C., 2009, MNRAS, 397, 1661 
Ventura P., D'Antona F., 2009, A\&A, 499, 835

Ventura P., Di Criscienzo M., Carini R., D'Antona F., 2013, MNRAS, 431, 3642

Villaume A., Conroy C., Johnson B. D., 2015, ApJ, 806, 82

Weiss A., Ferguson J. W., 2009, A\&A, 508, 1343

Weisz D. R., Dolphin A. E., Skillman E. D., Holtzman J., Gilbert K. M., Dalcanton J. J., Williams B. F., 2014, ApJ, 789, 147 Williams B. F., et al., 2017, ApJ, 846, 145

Yurchenko S. N., Szabó I., Pyatenko E., Tennyson J., 2018, MNRAS, 480, 3397

Zhukovska S., Henning T., 2013, A\&A, 555, A99

Zhukovska S., Gail H. P., Trieloff M., 2008, A\&A, 479, 453

Zibetti S., Gallazzi A., Charlot S., Pierini D., Pasquali A., 2013, MNRAS, 428, 1479

van Loon J. T., Zijlstra A. A., Kaper L., Gilmore G. F., Loup C., Blommaert J. A. D. L., 2001, A\&A, 368, 239

van Loon J. T., Marshall J. R., Zijlstra A. A., 2005, A\&A, 442,597 